\title{
On pseudospheres that are quasispheres
}

John L. Lewis and Andrew Vogel

Abstract. We construct bounded domains $D$ not equal to a ball in $n \geq 3$ dimensional Euclidean space, $\mathbb{R}^{n}$, for which $\partial D$ is homeomorphic to a sphere under a quasiconformal mapping of $\mathbb{R}^{n}$ and such that $n-1$ dimensional Hausdorff measure equals harmonic measure on $\partial D$.

\section{Introduction.}

Denote points in Euclidean space, $\mathbb{R}^{n}$, by $x=\left(x_{1}, \ldots, x_{n}\right)$ and let $\bar{E}, \partial E$, denote the closure and boundary of $E \subseteq \mathbb{R}^{n}$, respectively. Put $B(x, r)=\{y:|y-x|<r\}$ and $S(x, r)=\{y:|y-x|=r\}$ when $r>0$. Define $k$ dimensional Hausdorff measure, $1 \leq k \leq n$, in $\mathbb{R}^{n}$ as follows: For fixed $\delta>0$ and $E \subseteq \mathbb{R}^{n}$, let $L(\delta)=\left\{B\left(x_{i}, r_{i}\right)\right\}$ be such that $E \subseteq \cup B\left(x_{i}, r_{i}\right)$ and $0<r_{i}<\delta, i=1,2, \ldots$ Set

$$
\phi_{\delta}^{k}(E)=\inf _{L(\delta)}\left(\sum \alpha(k) r_{i}^{k}\right)
$$

where $\alpha(k)$ denotes the volume of the unit ball in $\mathbb{R}^{k}$. Then

$$
H^{k}(E)=\lim _{\delta \rightarrow 0} \phi_{\delta}^{k}(E), \quad 1 \leq k \leq n .
$$

Let $D$ be a bounded domain in $\mathbb{R}^{n}$ with $0 \in D$ and $H^{n-1}(\partial D)<+\infty$. 
Then $\partial D$ is said to be a pseudo sphere (see $[\mathrm{S}]$ ) if

a) $D \neq$ ball and there is a homeomorphism

$$
f: \mathbb{R}^{n} \longrightarrow \mathbb{R}^{n} \text { with } f(S(0,1))=\partial D,
$$

b) $h(0)=a \int_{\partial D} h d H^{n-1}$, whenever $h$ is harmonic

in $D$ and continuous on $\bar{D}$.

In b), a denotes a constant. The construction of pseudo spheres in $\mathbb{R}^{2}$, which are not circles, was first done by Keldysh and Lavrentiev to show the existence of domains not of Smirnov type (see [KL], [P, Chapter 3]). Also a completely different proof of existence in $\mathbb{R}^{2}$ has been given by Duren, Shapiro, and Shields in [DSS] (see also [Du, Chapter 10]). In higher dimensions we proved in [LV].

Theorem A. There exists a pseudo sphere in $\mathbb{R}^{n}, n \geq 3$.

Recall that a function $g: \mathbb{R}^{n} \longrightarrow \mathbb{R}^{n}$ is said to be $K \geq 1$ quasiconformal on $\mathbb{R}^{n}$ (see $[\mathrm{R}]$, [Re]) if:

i) $g$ is a homeomorphism of $\mathbb{R}^{n}$ onto $\mathbb{R}^{n}$,

ii) $g$ has distributional partial derivatives that are locally $n$-th power integrable,

iii) $\|D g(x)\|^{n} \leq K J_{g}(x)$, almost everywhere.

In iii), $D g(x)=\left(\partial g_{i}(x) / \partial x_{j}\right)$, is the Jacobian matrix of $g$ and $\|D g(x)\|$ is the norm of $D g(x)$ as a linear operator on $\mathbb{R}^{n}$. Also $J_{g}(x)$ (the Jacobian of $g$ at $x$ ) is the determinant of $D g(x)$. In [LV] we asked whether $f$ in the definition of a pseudosphere can also be chosen $K>1$ quasiconformal from $\mathbb{R}^{n}$ to $\mathbb{R}^{n}$ when $n \geq 3$. If so, then $\partial D$ is said to be a $K$ quasisphere. In $\mathbb{R}^{2}$ it follows easily from the geometric construction of Keldysh and Lavrentiev and the Ahlfors three point condition $[\mathrm{A}]$ that there exists pseudospheres which are quasispheres. The construction in [DSS] (see also [D, Chapter 10]) is also easily seen to produce pseudospheres that are quasispheres. In this note we answer our own conjecture by proving

Theorem 1. Given $K>1$ there exists a pseudo sphere in $\mathbb{R}^{n}, n \geq 3$, which is a $K$ quasisphere. 
We note that the only 1 quasiconformal maps of $\mathbb{R}^{n}$ are Möbius transformations. Also, it was shown by $[\mathrm{FL}]$ that convex domains satisfying some mild smoothness conditions cannot be pseudospheres. More generally, let $G$ be Green's function for a bounded domain $D$ with pole at 0 and suppose $B(0,2 s) \subset D$. Assume that

$$
\begin{aligned}
& +)|\nabla G| \leq M<\infty \text { in } D \backslash B(0, s), \\
& ++) H^{n-1}\left(\partial D \backslash \partial D^{*}\right)=0, \text { where } \\
& \partial D^{*}=\left\{x \in \partial D: \limsup _{r \rightarrow 0} r^{-n} \min \left\{H^{n}(B(x, r) \cap D),\right.\right. \\
& \left.\left.H^{n}(B(x, r) \backslash D)\right\}>0\right\} .
\end{aligned}
$$

In [LV1, Theorem 5] we showed that if $\mathrm{b}),+),++$ ) are valid, then $D$ must be a ball. Recall that $\partial D$ is said to be Ahlfors regular if for some $r_{0}>0$ and every $x \in \partial D$ we have $H^{n-1}(B(x, r) \cap \partial D) \approx r^{n-1}$ where $\approx$ means the two quantities are related by constants independent of $x$ and $r, 0<r \leq r_{0}$. This inequality and b) are easily seen to imply + ). Also if $D$ is an NTA domain in the sense of Jerison and Kenig [JK], then ++ ) is valid. We conclude that an NTA domain whose boundary is Ahlfors regular and satisfies b) must be a ball. So in particular if $f$ is a bilipschitz mapping of $\mathbb{R}^{n}$ with $f(S(0,1))=\partial D$ and b) holds, then $D=$ ball. Thus pseudospheres can be nice (quasispheres) but not too nice (Lipschitz).

To point out some of the differences between Theorem 1 and Theorem A we need to recall some details from [LV]. Suppose $a=1$ in the definition of a pseudosphere. To construct $D$, let $D_{0}=B\left(0, \rho_{0}\right)$ and let

$$
G_{0}(x)=(n(n-2) \alpha(n))^{-1}\left(|x|^{2-n}-\rho_{0}^{2-n}\right), \quad x \in B\left(0, \rho_{0}\right),
$$

be Green's function for $B\left(0, \rho_{0}\right)$, where $\rho_{0}$ is chosen so that if $x \in$ $\partial B\left(0, \rho_{0}\right)$, then

$$
\left|\nabla G_{0}(x)\right|=(n \alpha(n))^{-1} \rho_{0}^{1-n}=2 .
$$

By induction, if $D_{m}$ has been defined for $m=$ a nonnegative integer, we added certain smooth bumps to $\partial D_{m}$ to get $D_{m+1}$ with $D_{m} \subset$ $D_{m+1}$. Then $D=\bigcup D_{m}$. To obtain $f$ we modified the identity mapping slightly in a neighborhood of each bump, to get $h_{m+1}$ a homeomorphism from $\mathbb{R}^{n}$ into $\mathbb{R}^{n}$, with $h_{m+1}\left(\partial D_{m}\right)=\partial D_{m+1}, h_{m+1}\left(D_{m}\right)=D_{m+1}$, for $m=0,1, \ldots$ Put $h_{0}(x)=\rho_{0} x$ and set $f_{k}(x)=h_{k} \circ h_{k-1} \circ \cdots \circ$ 
$h_{0}(x)$. Then $f=\lim _{k \rightarrow \infty} f_{k}$ uniformly in $\mathbb{R}^{n}$. The problem with our construction in $[\mathrm{LV}]$ was that the distortion (i.e. $K$ ) could build up under successive iterations. In the present paper we overcome this difficulty by using the so called "mickey mouse" construction which is apparently due to Thurston (oral communication to the first author by Seppo Rickman). Under this construction $h_{m+1}$ is defined in such a way that it is 1 quasi-conformal (i.e. the restriction of a Möbius transformation) in a neighborhood of $H^{n-1}$ almost every point of $\partial D_{m}$. To get $D_{m+2}$ we then only allow bumps to be added that lie in the image of the above neighborhoods. It turns out for $H^{n-1}$ almost every point $x \in \mathbb{R}^{n}$ that we can arrange it so that all functions in the composition defining $f_{k}(x)$, with one exception, are 1 quasiconformal, while the remaining function can be chosen $K$ quasiconformal for fixed $K>1$. We note that the construction of a pseudosphere in $\mathbb{R}^{2}$ given in $[\mathrm{P}]$ also uses circles, but for a different reason. To carry out the above program we have had to overcome certain problems not encountered in [LV]. For example in this paper we added $C^{\infty}$ bumps to $\partial D_{m}$ and consequently were able to use Schauder type theorems to make the desired estimates on the Green's function of $D_{m+1}$. However, to get $h_{m+1}$, as above, we are forced to add non smooth spherical bumps to $\partial D_{m}$. Hence we have to argue that our earlier program can still be used. Also in [LV] we used an important lemma of Wolff [W, Lemma 2.7] for the Green's function of a domain obtained by adding a $C^{\infty}$ bump to a half space. Again we have to verify that Wolff's lemma remains valid for spherical bumps (whose radius is large). As for the proof of Theorem 1 we follow closely the proof of Theorem A in [LV] so the reader is advised to have this paper at hand. In Section 2 we discuss adding spherical bumps to a domain and show inequality (1.1) in [LV] (see (2.3)) is still valid. In Section 3 we use the "mickey mouse construction" to get $D$ and $f$. In Section 4 we add a spherical bump to a half space and show that the conclusion of Wolff's lemma remains true. We then use this lemma in Section 5 to show that (1.3) in [LV] (see (3.14)) still holds. (1.2), (1.3), and (1.9) of [LV] imply that (1.1) b) is valid (see the discussion in Section 3 following (3.11)).

\section{Spherical bumps.}

We assume throughout this section that $\Omega$ is a bounded domain with $0 \in \Omega$. Moreover we assume $\Omega$ is locally Lipschitz. That is given 
$y \in \partial \Omega$ there exists $s>0$ such that $B(y, s) \cap \partial \Omega$ is a part of the graph of a Lipschitz function defined on a hyperplane in $\mathbb{R}^{n}$ and $B(y, s) \cap \Omega$ lies above the graph. We also assume that $\partial \Omega$ is connected and the union of a finite number of closed spherical caps with centers in $\Omega$ and the property that each point of $\partial \Omega$ lies in at most two spherical caps. Thus either two caps are disjoint or their intersection is an $n-2$ dimensional "circle" (intuitively cut out by the smaller sphere from the larger sphere). Let $T$ denote the set of points in the union of these "circles". Finally we assume that $F \subset \mathbb{R}^{n}$ is a compact set with $F \cap \partial \Omega \subset T$. We remark that in our construction $F$ will be the set of points where a certain iterate is not 1 quasiconformal. Intuitively we want to avoid this set in modifying $\Omega$ to get $\Omega^{\prime}$ so that successive iterations will not increase $K$. Let $G$ be Green's function for $\Omega$ with pole at 0 . By definition,

$$
G(x)-(n(n-2) \alpha(n))^{-1}|x|^{2-n}, \quad x \in \mathbb{R}^{n},
$$

is harmonic in $\Omega$ and $G$ has boundary value 0 in the sense of PerronWiener-Brelot. Using the Kelvin transformation (see $[\mathrm{H}]$ ) we see that each component of

$$
\nabla G(x)=\left(\frac{\partial G}{\partial x_{1}}, \ldots, \frac{\partial G}{\partial x_{n}}\right)
$$

extends to a $C^{\infty}$ function on $\bar{\Omega} \backslash(T \cup\{0\})$. Under this assumption suppose that

$$
|\nabla G|>1, \quad \text { on } \partial \Omega \backslash T \text {. }
$$

Given $\delta, 0<\delta \leq 10^{-20}$, we shall add smooth spherical bumps to $\partial \Omega$ by "pushing out" $\partial \Omega$ along certain small surface elements in $\{x \in \partial \Omega \backslash T$ : $|\nabla G|(x)>1+\delta\}$ of approximate side length $r^{\prime}$. Let $\Omega^{\prime}, G^{\prime}$ be the domain and Green's function with pole at 0 , obtained from this process. Then $\partial \Omega^{\prime}$ will have the same properties as $\partial \Omega$, i.e. it is locally Lipschitz, connected, and the union of a finite number of closed spherical caps with centers in $\Omega^{\prime}$ and the property that each point of $\partial \Omega^{\prime}$ lies in at most two spherical caps. Define $T^{\prime}$ relative to $\Omega^{\prime}$ in the same way that $T$ was defined relative to $\Omega$. Then $\Omega \subset \Omega^{\prime}, T \subset T^{\prime}$ and we shall choose the spherical bumps so that

$$
\left|\nabla G^{\prime}\right|>1, \quad \text { on } \partial \Omega^{\prime} \backslash T^{\prime} .
$$


Also for $t \geq \delta$, we shall have

$$
H^{n-1}\left(\partial \Omega^{\prime}\right) \geq H^{n-1}(\partial \Omega)+\eta(t) H^{n-1}(\{x:|\nabla G(x)|>1+t\}),
$$

where $\eta$ is a nondecreasing positive function on $(0, \infty)$ which is independent of $\Omega, \Omega^{\prime}$.

Let $0<\sigma_{0}<10^{-3}$ be a small positive number to be chosen in Section 5 and let $l$ be the largest nonnegative integer such that $2^{-l} \sigma_{0}>$ $\delta>0$. Put $\sigma_{k}=2^{-k} \sigma_{0}$, for $k=0,1, \ldots$ and set

$$
\begin{gathered}
E_{k}=\left\{x \in \partial \Omega: 1+\sigma_{k}<|\nabla G(x)| \leq 1+\sigma_{k-1}\right\}, \quad 1 \leq k \leq l+1 \\
E_{0}=\left\{x \in \partial \Omega:|\nabla G(x)|>1+\sigma_{0}\right\}
\end{gathered}
$$

Let $d\left(E_{1}, E_{2}\right)$ denote the Euclidean distance between the sets $E_{1}, E_{2}$ and put

$$
U=\left\{y \in \partial \Omega: d(\{y\}, T)<10^{8} \widehat{r}_{0}\right\},
$$

where $\widehat{r}_{0}>0$ is so small that

$$
H^{n-1}\left(E_{k} \cap U\right) \leq \frac{1}{4} H^{n-1}\left(E_{k}\right), \quad \text { for } 0 \leq k \leq l+1,
$$

which is possible since $H^{n-1}(T)=0$. Next if $0<\widehat{r}_{1}<1$ is the smallest radius of the spheres whose caps form $\partial \Omega$ we also choose

$$
\widehat{r}_{0} \leq\left(\frac{\delta \widehat{r}_{1}}{10}\right)^{20}
$$

Let

$$
V=\left\{y \in \bar{\Omega}: d(\{y\}, T) \geq 10^{4} \widehat{r}_{0}\right\} \cap\left\{y \in \bar{\Omega}: d(\{y\},\{0\}) \geq \frac{\rho_{0}}{2}\right\},
$$

where $\rho_{0}$ is as in (1.2) and set

$$
M_{1}=\max _{x \in V} \sum\left|\partial_{\beta} G(x)\right|,
$$

where $\beta=\left(\beta_{1}, \beta_{2}, \ldots, \beta_{n}\right), 0 \leq|\beta| \leq 2$, is a multiindex and $\partial_{\beta}$ denotes the corresponding partial derivative with respect to $x^{\beta}, x \in V$. We first choose $r_{0}^{\prime}, 0<r_{0}^{\prime} \leq \widehat{r}_{0}$, so that

$$
r_{0}^{\prime} \leq \frac{1}{\left(10 n M_{1}\right)^{10}} .
$$


Given $y \in \partial \Omega \backslash U$ let $B\left(\bar{y}, \bar{r}_{0}\right)$ denote the reflection of $B\left(y, 10^{4} r_{0}^{\prime}\right)$ with respect to the sphere whose spherical cap $\subset \partial \Omega$ contains $y$. From our assumptions on $\partial \Omega$ we can choose $r_{0}^{\prime}>0$ so small that for any $y \in \partial \Omega \backslash U$

$$
\begin{aligned}
& B\left(y, 10^{4} r_{0}^{\prime}\right) \text { intersects exactly one spherical cap } \subset \partial \Omega, \\
& \text { and }\left(B\left(y, 10^{4} r_{0}^{\prime}\right) \cup B\left(\bar{y}, \bar{r}_{0}\right)\right) \cap F=\varnothing .
\end{aligned}
$$

From compactness and a standard covering argument it follows for each $r^{\prime}, 0<r^{\prime} \leq r_{0}^{\prime}$, that there exists, $y^{1}, y^{2}, \ldots, y^{N} \in \partial \Omega \backslash U$, such that

$$
\begin{aligned}
& \partial \Omega \backslash U \subseteq \bigcup_{i=1}^{N} B\left(y^{i}, 100 r^{\prime}\right) \cap \bar{\Omega} \subseteq V \\
& \text { and } B\left(y^{i}, 10 r^{\prime}\right) \cap B\left(y^{j}, 10 r^{\prime}\right)=\varnothing, \quad i \neq j .
\end{aligned}
$$

We now construct $\Omega^{\prime}$. Let $L$ be the set of all $y \in\left\{y^{i}\right\}_{1}^{N}$ for which

$$
B\left(y, 100 r^{\prime}\right) \cap\left(\bigcup_{k=0}^{l+1} E_{k}\right) \neq \varnothing .
$$

Let $\lambda_{k} \geq 2, k=0, \ldots$ be an increasing sequence of positive numbers to be specified later and set $r_{k}=r^{\prime} / \lambda_{k}$ for $k=0, \ldots, l+1$. For fixed $y \in L$, let $j=j(y)$ be the smallest nonnegative integer with

$$
B\left(y, 100 r^{\prime}\right) \cap E_{j} \neq \varnothing .
$$

We draw a sphere $S(\widetilde{y}, \widetilde{r})$ of radius $\widetilde{r}$, center $\widetilde{y} \in \Omega$ with the following properties

a) $S(\widetilde{y}, \widetilde{r}) \cap \partial \Omega=S\left(y, \sigma_{j}^{2} r_{j}\right) \cap \partial \Omega$,

b) The angle between the normals to $S(\widetilde{y}, \widetilde{r})$

and $\partial \Omega$ at points of intersection is $\sigma_{j}^{2}$,

c) $B(\widetilde{y}, \widetilde{r}) \subset \Omega \cup B\left(y, \sigma_{j}^{2} r_{j}\right)$.

Existence of $S(\widetilde{y}, \widetilde{r})$ as in (2.10) follows from (2.5) and elementary geometry. Define $\Omega^{\prime}$ by

i) $\Omega \backslash\left(\bigcup_{z \in L} B\left(z, \sigma_{j}^{2} r_{j}\right)\right)=\Omega^{\prime} \backslash\left(\bigcup_{z \in L} B\left(z, \sigma_{j}^{2} r_{j}\right)\right)$,

ii) $\partial \Omega^{\prime} \cap \bar{B}\left(y, \sigma_{j}^{2} r_{j}\right)=S(\widetilde{y}, \widetilde{r}) \backslash \Omega$ whenever $y \in\left\{y^{i}\right\}_{1}^{N}$,

iii) $\Omega^{\prime} \cap B\left(y, \sigma_{j}^{2} r_{j}\right)=B(\widetilde{y}, \widetilde{r}) \cap B\left(y, \sigma_{j}^{2} r_{j}\right)$. 
From $(2.10),(2.11)$, and (2.7) it is clear that $\partial \Omega^{\prime}$ is locally Lipschitz, connected, and the union of a finite number of closed spherical caps with centers in $\Omega^{\prime}$ and the property that each point of $\partial \Omega^{\prime}$ lies in at most two spherical caps. We now prove (2.2). If $x \in \partial \Omega^{\prime} \cap \partial \Omega$, then it follows from (2.1) and the Hopf boundary maximum principle that (2.2) is true. Otherwise, $x \in S(\widetilde{y}, \widetilde{r}) \cap\left(\partial \Omega^{\prime} \backslash T^{\prime}\right)$ for some $y \in\left\{y^{i}\right\}_{1}^{N}, S(\widetilde{y}, \widetilde{r})$, satisfying (2.7)-(2.11). Using (2.5), (2.10) a), b) and high school geometry it is easily seen for $\sigma_{0}$ small enough that

$$
\frac{\widetilde{r}}{2} \leq r_{j} \leq 2 \widetilde{r}
$$

From (2.12), (2.5) we deduce that $S(\widetilde{y}, \widetilde{r}) \cap \bar{\Omega} \subset V$ and thereupon from (2.6), (2.1) as well as Taylor's theorem with remainder that

$$
\left(1-\left(r_{0}^{\prime}\right)^{1 / 2}\right)|\nabla G(y)|\langle\nu, z-y\rangle \leq G(z),
$$

whenever $z \in S(\widetilde{y}, \widetilde{r})$ and $\langle\nu, z-y\rangle \geq 8 \sigma_{j}^{4} r_{j}$. Here $\nu$ denotes the inner unit normal to $\partial \Omega$ at $y$ and $\langle\cdot, \cdot\rangle$ denotes the inner product on $\mathbb{R}^{n}$. Let $\phi$ be a $C^{\infty}$ function on $\mathbb{R}$ with $\phi=0$ in $\left(-\infty, 8 \sigma_{j}^{4} r_{j}\right), \phi(x) \leq x$, for $x \geq 0$, with equality when $x \geq 16 \sigma_{j}^{4} r_{j}$ and

$$
\left(\sigma_{j}^{4} r_{j}\right)^{i-1}\left|\frac{d^{i}}{d x^{i}}(\phi(x)-x)\right| \leq 10^{4},
$$

whenever $x \geq 0$ and $0 \leq i \leq 2$. Let $h$ be the harmonic function in $B(\widetilde{y}, \widetilde{r})$ which is continuous in $\bar{B}(\widetilde{y}, \widetilde{r})$, with boundary values $h(x)=$ $\phi(\langle\nu, x-y\rangle)$ whenever $x \in S(\widetilde{y}, \widetilde{r})$. Let

$$
H(z)=h(\widetilde{y}+\widetilde{r} z)-\langle\nu, \widetilde{y}-y+\widetilde{r} z\rangle, \quad \text { for } z \in B(0,1) .
$$

Using (2.12) and Schauder type estimates (see [GT]), or direct estimates by way of the Poisson integral for $B(0,1)$ we find that

$$
|\nabla H|(z) \leq c(n) \widetilde{r} \sigma_{j}^{3 / 2}
$$

whenever $\widetilde{y}+\widetilde{r} z \in \partial \Omega^{\prime}$. Transferring back we get

$$
|\nabla h-\nu| \leq c(n) \sigma_{j}^{3 / 2}
$$

in $S(\widetilde{y}, \widetilde{r}) \cap \partial \Omega^{\prime}$, where $c(n) \geq 1$ as in the sequel is a constant which only depends on $n$, not necessarily the same at each occurence. Since $G \leq G^{\prime}$ in $\Omega$, we see from (2.13) and the boundary values of $h$ that

$$
\left(1-\left(r_{0}^{\prime}\right)^{1 / 2}\right)|\nabla G(y)| h \leq G^{\prime},
$$


on $S(\widetilde{y}, \widetilde{r})$. Using the Hopf boundary maximum principle and (2.14), (2.15) it follows that

$$
\left|\nabla G^{\prime}\right| \geq\left(1-\left(r_{0}^{\prime}\right)^{1 / 2}\right)\left(1-c(n) \sigma_{j}^{3 / 2}\right)|\nabla G(y)|
$$

on $S(\widetilde{y}, \widetilde{r}) \cap \partial \Omega^{\prime}$. Now from $(2.5),(2.6),(2.9)$ we deduce that

$$
|\nabla G(y)| \geq 1+\frac{\sigma_{j}}{2}
$$

Putting this inequality in (2.16) we see for $\sigma_{0}=\sigma_{0}(n)>0$ small enough that (2.2) is true for $x \in S(\widetilde{y}, \widetilde{r}) \cap \partial \Omega^{\prime}$. Hence (2.2) is true on $\partial \Omega^{\prime} \backslash T^{\prime}$.

Next we prove (2.3). To do this observe from (2.5) that since $r^{\prime} \ll \widehat{r}_{1}$ we have

$$
\begin{aligned}
& H^{n-1}(\partial \Omega\left.\cap B\left(y, \sigma_{j}^{2} r_{j}\right)\right) \\
& \leq \alpha(n-1)\left(\sigma_{j}^{2} r_{j}\right)^{n-1}-c(n) \widehat{r}_{1}^{-2}\left(\sigma_{j}^{2} r_{j}\right)^{n+1} .
\end{aligned}
$$

Note from (2.5), (2.12), and elementary trigonometry, that the solid angle $\theta$ subtended by $B(\widetilde{y}, \widetilde{r}) \cap \partial \Omega$ with respect to $\widetilde{y}$ satisfies

$$
\left|\theta-\sigma_{j}^{2}\right| \leq \frac{4 \widetilde{r} \sigma_{j}^{2}}{\widehat{r}_{1}}
$$

and

$$
\widetilde{r} \sin \theta \geq \sigma_{j}^{2} r_{j}-\frac{\left(\sigma_{j}^{2} r_{j}\right)^{3}}{100 \widehat{r}_{1}^{2}}
$$

for $\sigma_{0}=\sigma_{0}(n)>0$ small enough. Now using spherical coordinates and (2.18.a) it is easily seen that

$$
H^{n-1}(S(\widetilde{y}, \widetilde{r}) \backslash \Omega) \geq \alpha(n-1)\left(1+\frac{\sigma_{j}^{4}}{c(n)}\right)(\widetilde{r} \sin \theta)^{n-1} .
$$

From this inequality, (2.18.b), and once again (2.5) we conclude that

$$
H^{n-1}\left(\partial \Omega^{\prime} \cap B\left(y, \sigma_{j}^{2} r_{j}\right)\right) \geq\left(1+\frac{\sigma_{j}^{4}}{c(n)}\right) \alpha(n-1)\left(\sigma_{j}^{2} r_{j}\right)^{n-1}
$$


Combining (2.17), (2.19), and using $\lambda_{j} r_{j}=r^{\prime}$, we find for some $c(n) \geq 1$ that

$$
\begin{aligned}
H^{n-1}\left(\partial \Omega^{\prime} \cap B\right. & \left.\left(y, \sigma_{j}^{2} r^{\prime} / \lambda_{j}\right)\right) \\
& \geq\left(1+\frac{\sigma_{j}^{4}}{c(n)}\right) H^{n-1}\left(\partial \Omega \cap B\left(y, \sigma_{j}^{2} \frac{r^{\prime}}{\lambda_{j}}\right)\right) .
\end{aligned}
$$

Let $\eta(t)=\sigma_{i}^{2 n+2} \lambda_{i}^{1-n} / c_{1}(n)$ for $\sigma_{i+1} \leq t<\sigma_{i}, i=0,1, \ldots$ and set $\eta(t)=\sigma_{0}^{2 n+2} \lambda_{0}^{1-n} / c_{1}(n)$ for $t \geq \sigma_{0}$. Then from (2.20), (2.4), and (2.8) we conclude for $c_{1}(n)$ large enough that (2.3) is true for $t \geq \delta$.

\section{The Mickey mouse construction.}

We continue with the same notation introduced in sections 1-2. Let $\Omega, \Omega^{\prime}, y \in\left\{y^{i}\right\}_{1}^{N}, r^{\prime}, \widetilde{r}=\widetilde{r}(j), S(\widetilde{y}, \widetilde{r}), \lambda_{j}$, and $\sigma_{j}^{2}$ be as in (2.7)-(2.12). Suppose that $B\left(y, 100 r^{\prime}\right) \cap \partial \Omega \subset S\left(w, \rho^{*}\right)$ with $B\left(w, \rho^{*}\right) \subset \Omega$. Choose a Möbius transformation $L$ so that

$$
\begin{aligned}
& \text { a) } L\left(B\left(w, \rho^{*}\right)\right)=H=\left\{x \in \mathbb{R}^{n}: x_{2}>0\right\} \text {, } \\
& \text { ק) } L\left(S\left(w, \rho^{*}\right) \cap S(\widetilde{y}, \widetilde{r})\right)=\left\{x \in \mathbb{R}^{n}: x_{1}=x_{2}=0\right\} \text {, } \\
& \gamma) L(B(\widetilde{y}, \widetilde{r}))=\widetilde{H} \text { and } L(\widetilde{H} \backslash H) \subset\left\{x: x_{1}, x_{2}<0\right\} \text {, } \\
& \delta) \text { The angle between the normals to } H, \widetilde{H} \\
& \text { at points of } \widetilde{H} \cap H \text { is } \sigma_{j}^{2} \text {. }
\end{aligned}
$$

(3.1) is easily proven using (2.10) b), as well as the fact that Möbius transformations preserve angles and map balls into hyperplanes or balls (see [Re, Chapter 3]). We introduce polar coordinates $x_{1}=r \cos \theta$, $x_{2}=r \sin \theta, r \geq 0,0 \leq \theta<2 \pi$. If $x=\left(x_{1}, x_{2}, \ldots, x_{n}\right)$ we put $\widehat{x}=$ $\left(x_{3}, \ldots, x_{n}\right)$ and write $x=\left(x_{1}, x_{2}, \widehat{x}\right)$. Next we define a quasiconformal mapping $q$ of $\mathbb{R}^{n}$ as follows

$$
\begin{gathered}
q(x)=x, \text { when } 0 \leq \theta \leq \sigma_{j}^{2}, \\
q(x)=\left(r \cos \left(\lambda\left(\theta-\sigma_{j}^{2}\right)+\sigma_{j}^{2}\right), r \sin \left(\lambda\left(\theta-\sigma_{j}^{2}\right)+\sigma_{j}^{2}\right), \widehat{x}\right) \\
\text { for } \sigma_{j}^{2}<\theta \leq \pi-\sigma_{j}^{2} \text { with } \lambda=\left(\pi-\sigma_{j}^{2}\right) /\left(\pi-2 \sigma_{j}^{2}\right), \\
q(x)=\left(r \cos \left(\theta+\sigma_{j}^{2}\right), r \sin \left(\theta+\sigma_{j}^{2}\right), \widehat{x}\right), \text { for } \pi-\sigma_{j}^{2}<\theta \leq \pi+\sigma_{j}^{2},
\end{gathered}
$$




$$
\begin{aligned}
& q(x)=\left(r \cos \left(\lambda^{*}\left(\theta-\pi-\sigma_{j}^{2}\right)+\pi+2 \sigma_{j}^{2}\right),\right. \\
& \left.\qquad r \sin \left(\lambda^{*}\left(\theta-\pi-\sigma_{j}^{2}\right)+\pi+2 \sigma_{j}^{2}\right), \widehat{x}\right), \\
& \text { for } \pi+\sigma_{j}^{2}<\theta \leq 2 \pi-\sigma_{j}^{2}, \text { with } \lambda^{*}=\left(\pi-3 \sigma_{j}^{2}\right) /\left(\pi-2 \sigma_{j}^{2}\right), \\
& \qquad(x)=x, \text { for } 2 \pi-\sigma_{j}^{2}<\theta<2 \pi .
\end{aligned}
$$

From the above definition of $q$ we note that

i) $q$ maps $H$ onto $H \cup \widetilde{H}$,

ii) $q$ is the identity mapping on

$$
\left\{x: x_{1} / \sqrt{x_{1}^{2}+x_{2}^{2}} \geq \cos \left(\sigma_{j}^{2}\right)\right\},
$$

iii) $q$ is a rotation on $\left\{x: x_{1} / \sqrt{x_{1}^{2}+x_{2}^{2}} \leq-\cos \left(\sigma_{j}^{2}\right)\right\}$,

iv) $q$ is $1+10 \sigma_{j}^{2}$ quasiconformal on $\mathbb{R}^{n}$.

Put $g(x)=L^{-1} \circ q \circ L(x)$ when $x \in \mathbb{R}^{n}$. From (2.7), (2.12), (3.1) we note that if

$$
\widetilde{F}=L^{-1} \circ q\left(\left\{x:-\cos \left(\sigma_{j}^{2}\right) \leq \frac{x_{1}}{\sqrt{x_{1}^{2}+x_{2}^{2}}} \leq \cos \left(\sigma_{j}^{2}\right)\right\}\right),
$$

then

$$
\begin{aligned}
& \left(\widetilde{F} \cup g^{-1}(\widetilde{F})\right) \subset B\left(y, 10^{4} r_{0}^{\prime}\right) \cup B\left(\bar{y}, \bar{r}_{0}\right) \\
& \text { and }\left(\widetilde{F} \cup g^{-1}(\widetilde{F})\right) \cap F=\varnothing,
\end{aligned}
$$

where the last line follows from (2.7). From (3.2) we also conclude that

a) $g$ is the identity transformation

on the unbounded component $I$ of $\mathbb{R}^{n} \backslash g^{-1}(\widetilde{F})$,

b) $g$ is a Möbius transformation

on the bounded component $J$ of $\mathbb{R}^{n} \backslash g^{-1}(\widetilde{F})$,

c) $g$ is $1+10 \sigma_{j}^{2}$ quasiconformal on $\mathbb{R}^{n}$.

We do this construction for each $y \in\left\{y^{i}\right\}_{1}^{N}$ obtaining functions $g_{1}, \ldots$, $g_{N}$ and sets $\widetilde{F}_{1}, I_{1}, J_{1}, \ldots, \widetilde{F}_{N}, I_{N}, J_{N}$, corresponding to $y^{1}, y^{2}, \ldots, y^{N}$, 
in such a way that (3.3), (3.4) hold with $g=g_{i}, 1 \leq i \leq N$. Define $\Phi: \mathbb{R}^{n} \longrightarrow \mathbb{R}^{n}$ by

$$
\Phi(x)= \begin{cases}x, & \text { when } x \in I_{1} \cap I_{2} \cdots \cap I_{N}, \\ g_{i}(x), & \text { when } x \in g_{i}^{-1}\left(\widetilde{F}_{i}\right) \cup J_{i}, 1 \leq i \leq N\end{cases}
$$

We note that $\Phi$ is well defined since from (3.3), (2.8), and (2.12) it follows that the sets $g_{i}^{-1}\left(\widetilde{F}_{i}\right) \cup J_{i}, 1 \leq i \leq N$, are pairwise disjoint. Using this note and (3.5) we conclude that

*) $\Phi(\Omega)=\Omega^{\prime}$ and $\Phi$ is a $1+10 \sigma_{j}^{2}$ quasiconformal mapping of $\mathbb{R}^{n}$ onto $\mathbb{R}^{n}$,

$* *) \Phi \equiv$ a Möbius transformation in each component

$$
\begin{gathered}
\text { of } \mathbb{R}^{n} \backslash \Phi^{-1}(\widehat{F}) \text { where } \widehat{F}=\bigcup_{i=1}^{N} \widetilde{F}_{i}, \\
* * *)\left(\widehat{F} \cup \Phi^{-1}(\widehat{F})\right) \cap F=\varnothing \text { and } F^{\prime}=\widehat{F} \cup F \\
\quad \text { is compact with } F^{\prime} \cap \partial \Omega^{\prime} \subset T^{\prime} .
\end{gathered}
$$

We now construct $D, f$. Let $D_{0}=B\left(0, \rho_{0}\right)$ be as in Section 1 where $\rho_{0}$ is as in (1.2) and set $F=F_{0}=T=T_{0}=\varnothing$. Let $\delta=\delta_{0}=10^{-20} \sigma_{0}$ and put $\Omega=D_{0}$. We use the results in Section 2 to get $\Omega^{\prime}=D_{1}$ satisfying (2.2), (2.3) and $\Phi_{1}=\Phi$ satisfying (3.6) with $\Phi_{1}\left(D_{0}\right)=D_{1}$. Let $F_{1}=F^{\prime}, T_{1}=T^{\prime}$ be the sets obtained from this construction. We now proceed by induction. Suppose $D_{k}, \Phi_{k}, T_{k}, F_{k}$ have been constructed using the results in Section 2 for $m \geq 1$ with

$$
\begin{aligned}
& D_{k} \subset D_{k+1}, T_{k} \subset T_{k+1}, F_{k} \subset F_{k+1}, \\
& \text { and } F_{k+1} \cap \partial D_{k+1} \subset T_{k+1}, \text { for } 0 \leq k \leq m-1,
\end{aligned}
$$

in such a way that

$$
\begin{aligned}
&\left|\nabla G_{k}\right|>1, \quad \text { on } \partial D_{k} \backslash T_{k} \\
& H^{n-1}\left(\partial D_{k+1}\right) \geq H^{n-1}\left(\partial D_{k}\right) \\
&+\eta(t) H^{n-1}\left(\left\{x:\left|\nabla G_{k}(x)\right|>1+t\right\}\right),
\end{aligned}
$$


whenever $t \geq \delta_{k}=10^{-20} \sigma_{k}, 0 \leq k \leq m-1$. Here $G_{k}$ denotes the Green's function for $D_{k}$ with pole at 0 . We also assume that

$$
\begin{aligned}
& \text { (3.6) holds with } \Phi, F, F^{\prime} \text { replaced by } \Phi_{k+1}, F_{k}, F_{k+1} \text {, } \\
& \text { respectively, for } 0 \leq k \leq m-1 \text {. }
\end{aligned}
$$

We put $\Omega=D_{m}, F=F_{m}, T=T_{m}$, and note from the induction hypothesis, (3.7), that $F \cap \partial \Omega \subset T$. If $\delta=\delta_{m}=10^{-20} \sigma_{m}$, then we can apply the results in Section 2 to get $\Omega^{\prime}=D_{m+1}, T^{\prime}=T_{m+1}$, for which (3.8), (3.9) hold when $k=m+1$. Also using (3.6) we get $F^{\prime}=F_{m+1}$, $\Phi=\Phi_{m+1}$, satisfying (3.10) with $k=m$. By induction we conclude that (3.7)-(3.10) holds, for each nonnegative integer $k$.

Put $D=\bigcup_{0}^{\infty} D_{i}$. We note that $f_{m}=\Phi_{m} \circ \cdots \circ \Phi_{1}$ maps $D_{0}$ onto $D_{m}$. From (3.6) ***), (3.7), and (3.10) it is clear for given $x \in \mathbb{R}^{n} \backslash T_{m-1}$ that each function in the composition defining $f_{m}$, with at most one exception, is a Möbius transformation in a neighborhood of $x$. Moreover such an exception is $1+10 \sigma_{0}^{2}$ quasiconformal in a neighborhood of $x$. Thus $f_{m}$ is $1+10 \sigma_{0}^{2}$ quasiconformal on $\mathbb{R}^{n}$ and $f_{m}\left(D_{0}\right)=D_{m}$ for $m \geq 1$. Now $\left\{f_{m}\right\}_{1}^{\infty}$ is a locally bounded sequence of $1+10 \sigma_{0}^{2}$ quasiconformal mappings on $\mathbb{R}^{n}$, so a subsequence (see [Re, Chapter 9] or $[R$, Chapter 6]) of this sequence either converges uniformly to a $1+10 \sigma_{0}^{2}$ quasiconformal $\widehat{f}$ from $\mathbb{R}^{n}$ to $\mathbb{R}^{n}$ or to a constant. Clearly a constant is ruled out. Put $f(x)=\widehat{f}\left(\rho_{0} x\right)$. Then from our construction we conclude that $f(B(0,1))=D, f(S(0,1))=\partial D$. Thus if $10 \sigma_{0}^{2}<K$, then (1.1) a) in Theorem 1 is true.

For the reader's convenience we outline the proof of b) given in [LV]. Using (2.5)-(2.12) it is intuitively clear for $\sigma_{0}$ small enough that $D$ is NTA in the sense of Jerison and Kenig [JK] with constant 1000 (see [LV, Section 4] for details). Also from Green's theorem and (3.8) we see that

$$
H^{n-1}\left(\partial D_{k}\right) \leq \int_{\partial D_{k}}\left|\nabla G_{k}\right| d H^{n-1}=1 .
$$

From (3.11) we see that $D$ is of finite perimeter in the sense of Federer (see [GE]). Thus as $k \longrightarrow \infty$,

$$
\left.\left.H^{n-1}\right|_{\partial D_{k}} \longrightarrow H^{n-1}\right|_{\partial^{*} D}=\left.H^{n-1}\right|_{\partial D}
$$

Here the convergence is weak convergence as measures. Also $\partial^{*} D$ is the reduced boundary of $D$. To get the last inequality, we note that $\partial^{*} D$ 
agrees $H^{n-1}$ almost everywhere with the so called measure theoretic boundary of $D$, defined as the set of points where the Lebesgue lower $n$ densities of $D, \mathbb{R}^{n} \backslash D$ are positive. Using the fact that $D$ is NTA, it is easily seen that $\partial D$ equals the measure theoretic boundary of $D$. Hence (3.12) is true (for a more direct proof see [LV, Section 4]). Also observe from (3.9) that

$$
\lim _{k \rightarrow \infty} H^{n-1}\left(\left\{x \in \partial D_{k}:\left|\nabla G_{k}(x)\right|>1+\delta\right\}\right)=0,
$$

for each $\delta>0$, since otherwise we could use (3.9) and iteration to get a contradiction. Finally we shall show in Section 5 that

$$
\int_{D_{k}}\left|\nabla G_{k}\right| \log \left|\nabla G_{k}\right| d H^{n-1} \leq c<\infty, \quad \text { for } k=0,1, \ldots
$$

From (3.14) we deduce for $\alpha>1, k=0,1, \ldots$

$$
\log \alpha \int_{\left\{\left|\nabla G_{k}\right|>\alpha\right\}}\left|\nabla G_{k}\right| d H^{n-1} \leq c<+\infty .
$$

Let $g \geq 0$ be a harmonic function in $D$ which is continuous on $\bar{D}$. Then from (3.8), (3.12), and Green's theorem we get

$$
\begin{aligned}
g(0) & =\int_{\partial D_{k}} g\left|\nabla G_{k}\right| d H^{n-1} \\
& \geq \int_{\partial D_{k}} g d H^{n-1} \longrightarrow \int_{\partial D} g d H^{n-1}
\end{aligned}
$$

as $k \longrightarrow \infty$. To obtain the reverse inequality for fixed $\delta<10^{-3}$ and $\alpha>10^{3}$, put

$$
\begin{gathered}
P_{k}=\left\{x \in \partial D_{k}: 1 \leq\left|\nabla G_{k}(x)\right| \leq 1+\delta\right\}, \\
Q_{k}=\left\{x \in \partial D_{k}: 1+\delta<\left|\nabla G_{k}(x)\right| \leq \alpha\right\}, \\
L_{k}=\left\{x \in \partial D_{k}:\left|\nabla G_{k}(x)\right|>\alpha\right\},
\end{gathered}
$$

for $k=0,1,2, \ldots$ Then

$$
g(0)=\int_{\partial D_{k}} g\left|\nabla G_{k}\right| d H^{n-1}=\int_{P_{k}} \cdots+\int_{Q_{k}} \cdots+\int_{L_{k}} \cdots=I_{1}+I_{2}+I_{3} .
$$


Clearly,

$$
\left|I_{1}\right| \leq(1+\delta) \int_{\partial D_{k}} g d H^{n-1} .
$$

Also from (3.13) we find that

$$
\left|I_{2}\right| \leq \alpha\|g\|_{\infty} H^{n-1}\left(\left\{x \in \partial D_{k}: 1+\delta \leq\left|\nabla G_{k}\right|\right\}\right) \longrightarrow 0,
$$

as $k \longrightarrow \infty$. Here, $\|g\|_{\infty}$ denotes the maximum of $g$ in $\bar{D}$. Using (3.15) we get

$$
\left|I_{3}\right| \leq\|g\|_{\infty} \int_{\left\{\left|\nabla G_{k}\right|>\alpha\right\}}\left|\nabla G_{k}\right| d H^{n-1} \leq c(\log \alpha)^{-1}\|g\|_{\infty} .
$$

Letting $k \longrightarrow \infty$ we obtain from the above estimates and (3.12) that

$$
g(0) \leq(1+\delta) \int_{\partial D} g d H^{n-1}+c(\log \alpha)^{-1}\|g\|_{\infty} .
$$

Finally letting $\delta \longrightarrow 0, \alpha \longrightarrow \infty$, we have

$$
g(0) \leq \int_{\partial D} g d H^{n-1}
$$

In view of (3.16) we conclude that

$$
g(0)=\int_{\partial D} g d H^{n-1}
$$

when $g \geq 0$ is continuous on $\bar{D}$ and harmonic in $D$. From (3.17) with $g \equiv 1$ we note that, $H^{n-1}(\partial D)=1$. If $g_{1}$ is continuous on $\bar{D}$, harmonic in $D$, and $g_{1}-m \geq 0$ in $\bar{D}$, then from (3.17) and the above note we deduce

$$
g_{1}(0)=\left(g_{1}-m\right)(0)+m=\int_{\partial D}\left(g_{1}-m\right) d H^{n-1}+m=\int_{\partial D} g_{1} d H^{n-1}
$$

Finally from a simple barrier estimate it is easily seen that for each $y \in T_{1}$,

$$
\infty=\limsup _{x \rightarrow y}\left|\nabla G_{1}\right|(x) \leq \limsup _{x \rightarrow y}|\nabla G|(x) .
$$

From this inequality we conclude that $D \neq$ ball. 
Thus $D$ is a pseudosphere and Theorem 1 is true once we have proved (3.14).

\section{Lemma of Wolff.}

If $x=\left(x_{1}, \ldots, x_{n}\right) \in \mathbb{R}^{n}$ we write $x=\left(x^{\prime}, x_{n}\right)$, where $x^{\prime}=$ $\left(x_{1}, \ldots, x_{n-1}\right)$. For given $\varepsilon>0,0<\varepsilon<1 / 10$, define $\phi(\cdot, \varepsilon)$ on $\mathbb{R}^{n-1}$ by

$$
\phi\left(x^{\prime}, \varepsilon\right)=\left\{\begin{aligned}
\varepsilon^{-1}\left(1-\sqrt{1+\varepsilon^{2}\left(1-\left|x^{\prime}\right|^{2}\right.}\right) & \\
& \text { when } x^{\prime} \in \mathbb{R}^{n-1} \text { and }\left|x^{\prime}\right| \leq 1, \\
0, & \\
& \text { when } x^{\prime} \in \mathbb{R}^{n-1} \text { and }\left|x^{\prime}\right|>1 .
\end{aligned}\right.
$$

Put $K=\left\{x \in \mathbb{R}^{n}: x_{n}>0\right\}$ and set $K(\varepsilon)=\left\{x=\left(x^{\prime}, x_{n}\right) \in \mathbb{R}^{n}: x_{n}>\right.$ $\left.\phi\left(x^{\prime}, \varepsilon\right)\right\}$. We note that $\partial K(\varepsilon) \backslash \partial K$ consists of the part of the sphere with center $\left(0, \ldots, 0, \varepsilon^{-1}\right)$ and radius, $\sqrt{\varepsilon^{-2}+1}$ which lies outside $K$. Thus $K(\varepsilon)$ is obtained by adding a spherical bump to $K$. Let $g(\cdot, \varepsilon)$ be the Green's function for $K(\varepsilon)$ with pole at $\infty$. That is, $g(x, \varepsilon)-x_{n}$ is a bounded harmonic function in $K(\varepsilon)$ and $g(\cdot, \varepsilon)$ is continuous on $\bar{K}(\varepsilon)$ with $g(\cdot, \varepsilon) \equiv 0$ on $\partial K(\varepsilon)$. Set

$$
I(\varepsilon)=\int_{\partial K(\varepsilon)}|\nabla g(\cdot, \varepsilon)| \ln |\nabla g|(\cdot, \varepsilon) d H^{n-1}
$$

Next let $\widehat{\theta}\left(x^{\prime}\right)=\left(1-\left|x^{\prime}\right|^{2}\right)^{+}, x^{\prime} \in \mathbb{R}^{n-1}$, where $a^{+}=\max \{a, 0\}$. Let $\theta$ denote the bounded harmonic function on $K$ which is continuous on $\bar{K}$ with $\theta=\widehat{\theta}$ on $\partial K=\mathbb{R}^{n-1}$. Put

$$
\Lambda(\theta)=\int_{\mathbb{R}^{n-1}}\left(\left(\theta_{x_{n}}\right)^{3}-3\left|\nabla^{\prime} \theta\right|^{2} \theta_{x_{n}}\right) d H^{n-1},
$$

where $\nabla^{\prime}$ denotes the gradient in $x^{\prime}$ only. We prove

Lemma 4.1. If $\Lambda(\theta)>0$, then there exists $c^{*}=c^{*}(n) \geq 1$, such that $I(\varepsilon) \leq-\varepsilon^{3} \Lambda(\theta) / 100$, for $0<\varepsilon \leq c^{*}(n)^{-1} \min \{\Lambda(\theta), 1\}$.

Proof. The proof is essentially the same as [W, Lemma 2.12]. However this lemma was proved under the assumption that $\partial K(\varepsilon)$ is smooth $\left(C^{\infty}\right)$ where in our case $\partial K(\varepsilon)$ is just Lipschitz. Therefore we include 
some details. We shall show that $I$ has continuous fourth derivatives and $\left|I^{\prime \prime \prime \prime}\right| \leq c(n)$ on $\left(0, \varepsilon_{0}\right)$ for $\varepsilon_{0}=\varepsilon_{0}(n)>0$, sufficiently small. Also it will turn out that the derivatives of $I$ can be found by differentiating under the integral sign as in [W] and $I(0)=I^{\prime}(0)=I^{\prime \prime}(0)=0$, while $I^{\prime \prime \prime}(0)=-(1 / 8) \Lambda(\theta)$. Using Taylor's theorem with remainder we then get Lemma 4.1 .

To begin, let $y \in \partial K(\varepsilon)$ and suppose for some $r>0$ that $w$ is harmonic in $K(\varepsilon) \cap B(y, 2 r)$ with continuous boundary values zero on $\partial K(\varepsilon) \cap B(y, 2 r)$ and $|w| \leq M<\infty$ in $B(y, 2 r) \cap K(\varepsilon)$. From a barrier type argument we find for $0<\varepsilon \leq \varepsilon_{0} \leq 1 / 100$, sufficiently small, that

$$
|w|(x) \leq c(n) M\left(\frac{|x-y|}{r}\right)^{9 / 10},
$$

for $x \in K(\varepsilon) \cap B(y, r)$. With $\varepsilon_{0}$ now fixed let $g(\cdot, z, \varepsilon)$ denote Green's function for $K(\varepsilon)$ with pole at $z \in K(\varepsilon)$ for $0<\varepsilon \leq \varepsilon_{0}$. We note that $g(x, z, \varepsilon) \leq c(n)|x-z|^{2-n}$ since the righthand side is a constant multiple of the Green's function for $\mathbb{R}^{n}$. Let $S=\left\{\left(x^{\prime}, 0\right) \in \mathbb{R}^{n}:\left|x^{\prime}\right|=\right.$ $1\}$ and let $\widehat{x}, \widehat{z} \in \partial K(\varepsilon) \cap(B(0,2) \backslash S)$. Let $x \in B(\widehat{x},|1-| \widehat{x}|| / 2)$, $z \in B(\widehat{z},|1-| \widehat{z}|| / 2)$, with $|1-| \widehat{x}|| \leq|1-| \widehat{z}|| / 16$. Then from (4.2) with $r=|x-z| / 2, y=\widehat{x}, w=g(\cdot, z, \varepsilon)$, and the above note it follows that

$$
g(x, z, \varepsilon) \leq c|1-| x||^{9 / 10}|x-z|^{1-n+1 / 10} .
$$

Next suppose that $v$ is harmonic in $K(\varepsilon)$ with

$$
v(x)=\int_{\partial K(\varepsilon) \cap B(0,1)}(1-|z|)^{4 / 5}|\nabla g(x, z, \varepsilon)| d H^{n-1} z, \quad x \in K(\varepsilon),
$$

where derivatives of $g(\cdot, \cdot, \varepsilon)$ are with respect to $z$. Under these assumptions we prove for $x \in B(\widehat{x},|1-| \widehat{x}|| / 2) \cap K(\varepsilon)$, and $\widehat{x} \in \partial K(\varepsilon) \cap B(0,2)$ that there exists $\widehat{c}(n) \geq 1$ with

$$
|v(x)| \leq \widehat{c}(n)|1-| x||^{4 / 5} .
$$

Now from Green's formula,

$$
|v(x)| \leq c(n)|1-| x||^{4 / 5}+\int_{J}(1-|z|)^{4 / 5}|\nabla g(x, z, \varepsilon)| d H^{n-1} z
$$

where $J=\{z \in \partial K(\varepsilon) \cap B(0,1):|1-| x||<(1 / 100)(1-|z|)\}$. From the Kelvin transformation (see $[\mathrm{H}]$ ), it is easily seen that $g(x, \cdot, \varepsilon)$ extends 
to a harmonic function in $B(z,|1-| z||)$ whenever $x$ is not in this ball and $z \in \partial K(\varepsilon) \backslash S$. We shall also denote this extension by $g(x, \cdot, \varepsilon)$. Using this fact, (4.3), and interior estimates for harmonic functions we see that

$$
|\nabla g(x, z, \varepsilon)| \leq c(n)|1-| x||^{9 / 10}|x-z|^{-n+1 / 10}, \quad \text { whenever } z \in J .
$$

Putting this estimate in (4.5), using $2|x-z| \geq|1-| z||$ when $z \in J$ and integrating we get (4.4).

Again from the Kelvin transformation, (4.2) with $w=g$, and interior estimates for harmonic functions we observe that

$$
\left|\frac{\partial^{k} g}{\partial x^{\alpha}}\right|(x, \varepsilon) \leq c(k, n)|1-| x||^{-k+9 / 10}, \quad x \in B\left(\widehat{x}, \frac{1}{2}|1-| \widehat{x}||\right),
$$

whenever $\widehat{x} \in \partial K(\varepsilon) \cap(B(0,2) \backslash S), 0<\varepsilon \leq \varepsilon_{0}$, and $k=0,1, \ldots$ Here $\alpha=\left(\alpha_{1}, \ldots, \alpha_{n}\right)$ is a multi index with $|\alpha|=k$ and $x^{\alpha}=x_{1}^{\alpha_{1}} \cdots x_{n}^{\alpha_{n}}$. Also we have

$$
g\left(x^{\prime}, \phi\left(x^{\prime}, \varepsilon\right), \varepsilon\right) \equiv 0, \quad x^{\prime} \in \mathbb{R}^{n-1}, \quad 0<\varepsilon \leq \varepsilon_{0} .
$$

Next observe for $k=0,1, \ldots$, that $\partial^{k} \phi(\cdot, \varepsilon) / \partial \varepsilon^{k}$ is uniformly Lipschitz for $x^{\prime} \in \mathbb{R}^{n-1}, 0<\varepsilon \leq \varepsilon_{0}$, with

$$
\begin{aligned}
& \text { i) }\left|\nabla \frac{\partial^{k} \phi\left(x^{\prime}, \varepsilon\right)}{\partial \varepsilon^{k}}\right| \leq c(n, k), \\
& \text { ii) }\left|\frac{\partial^{k} \phi\left(x^{\prime}, \varepsilon\right)}{\partial \varepsilon^{k}}\right| \leq c(n, k)\left(1-\left|x^{\prime}\right|\right)^{+}, \\
& \text {iii) } \frac{\partial \phi\left(x^{\prime}, 0\right)}{\partial \varepsilon}=-\frac{\left(1-\left|x^{\prime}\right|^{2}\right)^{+}}{2}, \frac{\partial^{2} \phi\left(x^{\prime}, 0\right)}{\partial \varepsilon^{2}} \equiv 0 .
\end{aligned}
$$

We claim that (4.6)-(4.8) imply $g(\cdot, \varepsilon)$ has continuous mixed partials in $x, \varepsilon$ of all orders whenever, $x \in B(\widehat{x},|1-| \widehat{x}|| / 2), 0<\varepsilon \leq \varepsilon_{0}$ and $\widehat{x} \in \partial K(\varepsilon) \backslash S$. Moreover if also $|\widehat{x}|<2$,

$$
\left|\frac{\partial^{k+l} g(x, \varepsilon)}{\partial x^{\alpha} \partial \varepsilon^{l}}\right| \leq c(k, l, n)|1-| x||^{-k+4 / 5},
$$

for $k, l=0,1, \ldots$, while

$$
\left|\frac{\partial^{k+l}\left(g(x, \varepsilon)-x_{n}\right)}{\partial x^{\alpha} \partial \varepsilon^{l}}\right| \leq c(k, l, n)|1+| x||^{-k-n+1},
$$


with $x \in \partial K(\varepsilon),|x| \geq 2$. (4.9) for $l=0, k=0,1, \ldots$ is implied by (4.6). (4.10) follows from the fact that $g(\cdot, \varepsilon)-x_{n}$ extends to a bounded harmonic function in $\mathbb{R}^{n} \backslash B(0,3 / 2)$ which is zero on $\left\{\left(x^{\prime}, 0\right): x^{\prime} \in\right.$ $\left.\mathbb{R}^{n-1},\left|x^{\prime}\right|>3 / 2\right\}$ and the Poisson integral formula for such functions. Thus (4.9), (4.10) are true for $l=0, k=0,1, \ldots$ We can now proceed by induction to get (4.9), (4.10). We do only the case $l=1, k=0, \ldots$, in detail. From (4.7), (4.8) ii), the mean value theorem from elementary calculus, and (4.6) for $k=1$ we see that

$$
\begin{aligned}
&\left|g\left(x^{\prime}, \phi\left(x^{\prime}, \varepsilon_{2}\right), \varepsilon_{2}\right)-g\left(x^{\prime}, \phi\left(x^{\prime}, \varepsilon_{2}\right), \varepsilon_{1}\right)\right| \\
&=\left|g\left(x^{\prime}, \phi\left(x^{\prime}, \varepsilon_{2}\right), \varepsilon_{1}\right)-g\left(x^{\prime}, \phi\left(x^{\prime}, \varepsilon_{1}\right), \varepsilon_{1}\right)\right| \\
& \leq c(n) \max \left\{\left|\frac{\partial g}{\partial x_{n}}\right|\left(x^{\prime}, \phi\left(x^{\prime}, \varepsilon\right), \varepsilon\right): 0<\varepsilon \leq \varepsilon_{0}\right\} \\
& \cdot\left|\phi\left(x^{\prime}, \varepsilon_{2}\right)-\phi\left(x^{\prime}, \varepsilon_{1}\right)\right| \\
& \leq c(n)\left|\varepsilon_{2}-\varepsilon_{1}\right|\left(\left(1-\left|x^{\prime}\right|\right)^{+}\right)^{4 / 5},
\end{aligned}
$$

for $x^{\prime} \in \partial K\left(\varepsilon_{2}\right) \backslash S, 0<\varepsilon_{1}, \varepsilon_{2} \leq \varepsilon_{0}$. From (4.11) we deduce that $\left\{\left(\varepsilon_{2}-\varepsilon_{1}\right)^{-1}\left(g\left(\cdot, \varepsilon_{2}\right)-g\left(\cdot, \varepsilon_{1}\right)\right)\right\}$ is uniformly bounded and has a continuous extension to $\partial K\left(\varepsilon_{2}\right)$ whenever $0<\varepsilon_{1}, \varepsilon_{2} \leq \varepsilon_{0}$ and $\varepsilon_{1} \neq \varepsilon_{2}$. From the maximum principle for harmonic functions and the Kelvin transformation, it follows that this sequence is harmonic and uniformly bounded in $L\left(\varepsilon_{2}\right)=K\left(\varepsilon_{2}\right) \bigcup\left\{B(\widehat{x},|1-| \widehat{x}||): \widehat{x} \in \partial K\left(\varepsilon_{2}\right) \backslash S\right\}$. Letting $\varepsilon_{1} \longrightarrow \varepsilon_{2}$ it follows that $\partial g / \partial \varepsilon$ is uniformly continous and bounded in $L\left(\varepsilon_{2}\right)$ whenever $0 \leq \varepsilon_{2} \leq \varepsilon_{0}$. Moreover,

$$
\frac{\partial g}{\partial \varepsilon}(x, \varepsilon)=-\frac{\partial g}{\partial x_{n}}(x, \varepsilon) \frac{\partial \phi}{\partial \varepsilon}\left(x^{\prime}, \varepsilon\right),
$$

with $x=\left(x^{\prime}, \phi\left(x^{\prime}, \varepsilon\right)\right) \in \partial K(\varepsilon) \backslash S$. Using (4.8) ii) and (4.6) with $k=1$ we get $|\partial g / \partial \varepsilon(\cdot, \varepsilon)| \leq c(n)\left((1-|x|)^{+}\right)^{4 / 5}$ on $\partial K(\varepsilon)$. Using this inequality and the maximum principle for bounded harmonic functions in $K(\varepsilon)$ we conclude first that $|\partial g / \partial \varepsilon| \leq c(n) v$, and thereupon from (4.4), the Kelvin transformation, and interior estimates for harmonic functions that (4.9) is true when $l=1, k=0,1, \ldots$ (4.10) follows for $l=1$ by the same reasoning as when $l=0$. Finally since a uniformly convergent sequence of harmonic functions has derivatives which also converge uniformly, it follows that the mixed partial derivatives consisting of one partial derivative in $\varepsilon$ and $k$ partial derivatives in the space variable $x$ are independent of the order of differentiation. 
Next we use (4.12) and argue as in (4.11) to obtain that

$$
\begin{aligned}
\frac{\partial^{2} g}{\partial \varepsilon^{2}}(x, \varepsilon)= & -2 \frac{\partial^{2} g}{\partial \varepsilon \partial x_{n}}(x, \varepsilon) \frac{\partial \phi}{\partial \varepsilon}\left(x^{\prime}, \varepsilon\right) \\
& -\frac{\partial g}{\partial x_{n}}(x, \varepsilon) \frac{\partial^{2} \phi}{\partial \varepsilon^{2}}\left(x^{\prime}, \varepsilon\right)-\frac{\partial^{2} g}{\partial x_{n}^{2}}(x, \varepsilon)\left(\frac{\partial \phi}{\partial \varepsilon}\right)^{2}\left(x^{\prime}, \varepsilon\right),
\end{aligned}
$$

whenever $x \in \partial K(\varepsilon)$. Using (4.8) ii) and (4.9) with $l=0,1, k=1,2$, we conclude first that $\left|\partial^{2} g / \partial \varepsilon^{2}(x, \varepsilon)\right| \leq c(n)\left((1-|x|)^{+}\right)^{4 / 5}$ when $x \in \partial K(\varepsilon)$ and thereupon from (4.4), the Kelvin transformation, and interior estimates for harmonic functions, that (4.9) is true when $l=0,1,2$. (4.10) follows by the same reasoning as when $l=0,1$. As above we see that the mixed partial derivatives consisting of two partial derivatives in $\varepsilon$ and $k$ partial derivatives in the space variable $x$ are independent of the order of differentiation. Continuing by induction we get (4.9), (4.10).

Finally observe from a barrier argument that

$$
c(n)|\nabla g(\cdot, \varepsilon)| \geq 1, \quad \text { on } \partial K(\varepsilon) \backslash S,
$$

for $0<\varepsilon \leq \varepsilon_{0}$. Using (4.9), (4.10), (4.14) we deduce that derivatives of $I$ with respect to $\varepsilon$ of all orders can be found by differentiating under the integral sign defining $I$. Doing this and letting $\varepsilon \longrightarrow 0$ we find that the argument of Wolff [W, pp. 360-362] can be used essentially verbatim. One only needs to check that the second and third partial derivatives of $\phi$ with respect to $\varepsilon$ do not add additional terms in the calculations when $\varepsilon=0$. In fact from (4.8) iii) we see that the second partial of $\phi$ with respect to $\varepsilon$ vanishes identically. Moreover all terms involving the third partial of $\phi$ with respect to $\varepsilon$ vanish at $\varepsilon=0$ (since all second partials of $g(x, 0)=x_{n}$ are identically zero and $\left.|\nabla g(x, 0)| \equiv 1\right)$. Lemma 4.1 now follows from Wolff's argument in the way mentioned at the beginning of the proof.

In order to apply Wolff's lemma we need to show that $\Lambda(\theta)>0$. In fact we shall show in [LVV] that if $\widehat{\psi} \geq 0$, is a radial, nonincreasing, Lipschitz function on $\mathbb{R}^{n-1}$ with compact support and $\widehat{\psi} \not \equiv 0$, then $\Lambda(\psi)>0$. As usual, $\psi$ denotes the bounded harmonic extension of $\widehat{\psi}$ to $K$ which is continuous on $\bar{K}$ with $\psi=\widehat{\psi}$ on $\partial K$. Clearly this result implies

$$
\Lambda(\theta)>0 .
$$

Here we outline a direct method for establishing (4.15) which gives a numerical lower bound for the integral when $n \geq 5$. Using separation 
of variables or the Poisson integral formula for harmonic functions in a half space one can show for $r=\left|x^{\prime}\right|$ that

$$
\theta_{x_{n}}\left(x^{\prime}, 0\right)=-c_{n} F\left(\frac{n}{2},-\frac{1}{2}, \frac{n-1}{2}, r^{2}\right), \quad 0<r<1,
$$

where $F(a, b, c, z)$ is the usual hypergeometric function,

$$
c_{n}=\frac{2 \Gamma\left(\frac{n}{2}\right)}{\Gamma\left(\frac{n-1}{2}\right) \Gamma\left(\frac{3}{2}\right)},
$$

and $\Gamma$ is the Euler gamma function. Writing the hypergeometric function in a series it is easily seen that

$$
-F\left(\frac{n}{2},-\frac{1}{2}, \frac{n-1}{2}, r^{2}\right) \geq-1+\frac{n}{n-1}\left(1-\left(1-r^{2}\right)^{1 / 2}\right) .
$$

Using this estimate and doing some arithmetic we find that

$$
\begin{aligned}
\int_{0}^{1} \theta_{x_{n}}^{3} r^{n-2} d r \geq & c_{n}^{3}(n-1)^{-3} \int_{0}^{1}\left(-1+n\left(1-r^{2}\right)^{1 / 2}\right)^{3} r^{n-2} d r \\
= & c_{n}^{3}(n-1)^{-3}\left(\frac{1}{n-1}+\frac{6 n^{2}}{n^{2}-1}\right) \\
& -6 c_{n}^{2}(n-1)^{-3}\left(1+\frac{n^{2}}{n+2}\right) \\
= & b_{n} .
\end{aligned}
$$

Also we note that

$$
\begin{aligned}
3 \int_{0}^{1} \theta_{x_{n}}\left|\nabla^{\prime} \theta\right|^{2} r^{n-2} d r & =-12 c_{n} \int_{0}^{1} F\left(\frac{n}{2},-\frac{1}{2}, \frac{n-1}{2}\right) r^{n} d r \\
& =\frac{48(n-2)}{(n+2) \pi n} .
\end{aligned}
$$

The last equality is obtained by writing out the series for the integrand and integrating term by term. The series one gets after evaluating at 1 can be written as the sum of several hypergeometric functions evaluated at 1 . Using tables one then gets the last equality. Finally using Stirling's formula and making some more estimates one can show 
that $b_{n}+48(n-2) /((n+2) \pi n) \geq 0$ for $n \geq 5$, which in view of (4.16), the above equality, and the fact that $\theta_{x_{n}}(r, 0) \geq 0$ for $r \in(1, \infty)$ (by positivity of $\theta$ ) implies (4.15) for $n \geq 5$. The cases $n=3,4$ can be done separately. A more involved argument using estimates also for $\int_{1}^{\infty} \theta_{x_{n}}^{3} r^{n-2} d r$ can be used to show that for some absolute constant $c$ one has $\Lambda(\theta) \geq c / n, n=3,4, \ldots$ (more details will be supplied upon request).

Next we introduce some notation in order to state some consequences of Lemma 4.1 and (4.15). Let $\Omega_{1}$ be a bounded domain with diameter $\approx 1$ and NTA constant 1000 . Then by definition,

i) (corkscrew condition) For each $x \in \partial \Omega_{1}$,

$0<r<1$, there are points $P_{r}(x) \in \Omega_{1}, Q_{r}(x) \in \mathbb{R}^{n} \backslash \Omega_{1}$,

with $\left|P_{r}(x)-x\right| \leq 1000 r,\left|Q_{r}(x)-x\right| \leq 1000 r$, and

$\operatorname{dist}\left(P_{r}(x), \partial \Omega_{1}\right) \geq 1000^{-1} r, \operatorname{dist}\left(Q_{r}(x), \partial \Omega_{1}\right) \geq 1000^{-1} r$,

ii) (Harnack chain condition) For each $x, y \in \Omega_{1}$ there is a path

$\gamma:[0,1] \longrightarrow \Omega_{1}$ with $\gamma(0)=x, \gamma(1)=y$, and with

length $\leq 1000|x-y|$. Also

$\operatorname{dist}\left(\gamma(t), \partial \Omega_{1}\right) \geq 1000^{-1} \min \{|\gamma(t)-x|,|\gamma(t)-y|\}$ for $t \in[0,1]$.

Next suppose that $\Omega_{1}$ is Lipschitz on scale $t$ with constant 1000 . That is assume for each $z \in \partial \Omega_{1}$, there exists a coordinate system such that $\partial \Omega_{1} \cap B(z, t)$ is the graph of a Lipschitz function defined on $\mathbb{R}^{n-1}$ with Lipschitz norm $\leq 1000$. Moreover, $\Omega_{1} \cap B(z, t)$ lies above the graph of this function. Finally assume for some $w \in \partial \Omega_{1}$ and $t>0$ that after a possible rotation of coordinates,

$$
\begin{aligned}
& \partial \Omega_{1} \cap B(w, t)=\left\{x: x_{n}=w_{n}\right\} \cap B(w, t) \\
& \Omega_{1} \cap B(w, t)=\left\{x: x_{n}>w_{n}\right\} \cap B(w, t) .
\end{aligned}
$$

Let $\phi(\cdot, \varepsilon)$ be as defined at the beginning of Section $4, \lambda \geq 2$, and define $\Omega_{2}(\varepsilon) \supset \Omega_{1}$ for $0<\varepsilon \leq \varepsilon_{0}$, as follows:

a) $\Omega_{1} \backslash B(w, t)=\Omega_{2}(\varepsilon) \backslash B(w, t)$,

b) $\partial \Omega_{2}(\varepsilon) \cap B(w, t)=\left\{\left(x^{\prime}+w^{\prime}, w_{n}+t \lambda^{-1} \phi\left(t^{-1} \lambda x^{\prime}, \varepsilon\right)\right): x^{\prime} \in\right.$ $\left.\mathbb{R}^{n-1}\right\} \cap B(w, t)$, 

$B(w, t)$.

c) $\Omega_{2}(\varepsilon) \cap B(w, t)=\left\{\left(x^{\prime}+w^{\prime}, x_{n}\right): x_{n}>w_{n}+t \lambda^{-1} \phi\left(t^{-1} \lambda x^{\prime}, \varepsilon\right)\right\} \cap$

We assume

$$
B\left(0, \rho_{0}\right) \subseteq \Omega_{1} \subseteq B(0,1)
$$

where $\rho_{0}$ is as in (1.2). Denote Green's functions for $\Omega_{1}, \Omega_{2}(\varepsilon)$, with pole at 0 , by $G_{1}, G_{2}(\cdot, \varepsilon)$ respectively, and let $\omega_{1}$ be harmonic measure on $\Omega_{1}$ with respect to 0 . With this notation we state

Lemma 4.19. Let $\Omega_{1}$ be NTA and Lipschitz on scale $t$ with constant 1000. Suppose $\Omega_{1}$ satisfies (4.17), (4.18), and $\Omega_{2}$ is obtained by adding a spherical bump to $\Omega_{1}$ as in a)-c). Let $\varepsilon_{0}=\left(2 c^{*}(n)\right)^{-1} \min \{\Lambda(\theta), 1\}$, where $c^{*}$ is as in Lemma 4.1. If $0<\widehat{\varepsilon} \leq \varepsilon_{0}$, then there exists $\lambda^{*}=$ $\lambda^{*}(\widehat{\varepsilon}, n), \bar{c}=\bar{c}(\widehat{\varepsilon}, n) \geq 2$, such that for $\lambda \geq \lambda^{*}$,

$$
\begin{aligned}
\int_{\partial \Omega_{2}(\varepsilon)}\left|\nabla G_{2}(\cdot, \varepsilon)\right| \log \left|\nabla G_{2}\right|(\cdot, \varepsilon) d H^{n-1} \\
\quad \leq \int_{\partial \Omega_{1}}\left|\nabla G_{1}\right| \log \left|\nabla G_{1}\right| d H^{n-1}-\frac{1}{\bar{c} \lambda^{n-1}} \omega_{1}(B(w, t))
\end{aligned}
$$

whenever $\widehat{\varepsilon} \leq \varepsilon \leq \varepsilon_{0}$.

Proof. In view of Lemma 4.1 and (4.15) we can essentially apply [W, Lemma 2.7] to get Lemma 4.19 in $\mathbb{R}^{3}$. The proof in $\mathbb{R}^{n}, n>3$, is unchanged.

\section{Proof of Theorem 1.}

Armed with Lemma 4.19 we can use the argument in [LV, Section 3] to prove (3.14) and hence complete the proof of Theorem 1. Unfortunately, in [LV, Section 3] Schauder estimates for smooth domains were again used, whereas our boundaries are only locally Lipschitz. Thus for the reader's convenience we sketch the argument in [LV, Section 3] indicating the necessary changes. We wish to apply Lemma 4.19 to $D_{m}, D_{m+1}$ constructed in Section 3 , but in order to do so we need to introduce intermediary domains with flat bumps as in Lemma 4.19 and make some estimates. We shall use the same notation as in Section 2. Note that in Section 3 we constructed $D_{m+1}$ from $D_{m}$ by adding 
spherical bumps as in (2.9)-(2.11). Thus we work with $\Omega, \Omega^{\prime}$ as in Section 2. We assume, as we may, that $\sigma_{0}^{2} \leq \varepsilon_{0} / 100$ where $\sigma_{0}$ is yet to be fixed and $\varepsilon_{0}$ is as in Lemma 4.19. We now define $\left(\lambda_{k}\right)$ introduced above (2.9). Let $\widehat{r}_{0}, \widehat{r}_{1}, \delta, M_{1}, r_{0}^{\prime}, r^{\prime},\left(E_{k}\right), L, l, r_{j}$ be as in Section 2. For fixed $y \in L$ recall from (2.9) that $j$ was the least postive integer such that $B\left(y, 100 r^{\prime}\right) \cap E_{j} \neq \varnothing, 1 \leq j \leq l+1$. Let $T$ be the tangent plane to $\partial \Omega$ at $y \in \partial \Omega$. From the above restriction on $\sigma_{0},(2.5)$, we see as in (2.18) a) that the central angle, say $\eta_{j}=\eta_{j}(y)$, subtended by $B(\widetilde{y}, \widetilde{r}) \cap T$ (relative to $\widetilde{y}$ ) satisfies $2^{-1} \sigma_{j}^{2} \leq \eta_{j} \leq 2 \sigma_{j}^{2} \leq \varepsilon_{0} / 4$, regardless of the choice of $\lambda_{j} \geq 2, y \in L$ or $r^{\prime}$. Put $\varepsilon_{j}=\tan \left(\sigma_{j}^{2} / 2\right), \lambda_{j}^{*}=\lambda^{*}\left(\varepsilon_{j}, n\right)$ and set $\lambda_{j}^{\prime}=\max \left\{\sigma_{j}^{-2}, b_{j}, \lambda_{j}^{*}\right\}, j=0,1, \ldots$, where $b_{j}=\bar{c}\left(\varepsilon_{j}, n\right)$. Let $\lambda_{k}=\max _{0 \leq j \leq k} \lambda_{j}^{\prime}, k=0,1, \ldots$, and observe that $\left(\lambda_{k}\right)_{0}^{\infty}$ depends only on $n$ once $\sigma_{0}$ is fixed.

We add flat bumps to $\Omega, \Omega^{\prime}$ as follows. Let $y, j$ be as above and as in (3.1) let $S\left(w, \rho^{*}\right)$ be such that $B\left(y, 100 r^{\prime}\right) \cap \partial \Omega \subset S\left(w, \rho^{*}\right)$ and $B\left(w, \rho^{*}\right) \subset \Omega$. After a rotation if necessary we may assume that $y=$ $\left(w^{\prime}, w_{n}-\rho^{*}\right)$, where $w=\left(w^{\prime}, w_{n}\right)$. Let $A=\rho^{*}-\sqrt{\left(\rho^{*}\right)^{2}-\left(r^{\prime}+\left(r^{\prime}\right)^{3 / 2}\right)^{2}}$ and define $\psi: \mathbb{R}^{n-1} \longrightarrow \mathbb{R}$ by

$$
\psi\left(x^{\prime}\right)=\left\{\begin{array}{cc}
w_{n}-\rho^{*}, & \text { for }\left|x^{\prime}-w^{\prime}\right| \leq r^{\prime}, \\
\left(r^{\prime}\right)^{-3 / 2} A\left(\left|x^{\prime}-w^{\prime}\right|-r^{\prime}\right)+w_{n}-\rho^{*}, & \text { for } r^{\prime} \leq\left|x^{\prime}-w^{\prime}\right| \leq r^{\prime}+\left(r^{\prime}\right)^{3 / 2}, \\
w_{n}-\sqrt{\left(\rho^{*}\right)^{2}-\left|x^{\prime}-w^{\prime}\right|^{2}}, \\
\text { for } r^{\prime}+\left(r^{\prime}\right)^{3 / 2} \leq\left|x^{\prime}-w^{\prime}\right| \leq \rho^{*} .
\end{array}\right.
$$

Note that the graph of $\psi$ coincides with the tangent plane $T$ to $S\left(w, \rho^{*}\right)$ at $y$ when $\left|x^{\prime}-w^{\prime}\right| \leq r^{\prime}$ and "linearly" connects this tangent plane with $S\left(w, \rho^{*}\right)$ when $r^{\prime} \leq\left|x^{\prime}-w^{\prime}\right| \leq r^{\prime}+\left(r^{\prime}\right)^{3 / 2}$. Suppose that $L=$ $\left\{z_{1}, z_{2}, \ldots, z_{p}\right\}$ and put $L_{k}=\left\{z_{1}, \ldots, z_{k}\right\}, 1 \leq k \leq p$.

Define $\widehat{\Omega}_{k}, 1 \leq k \leq p$, by

$$
\begin{aligned}
& \widehat{\mathrm{I}}) \widehat{\Omega}_{k} \backslash\left(\bigcup_{z \in L_{k}} B\left(z, 10 r^{\prime}\right)\right)=\Omega \backslash\left(\bigcup_{z \in L_{k}} B\left(z, 10 r^{\prime}\right)\right), \\
& \widehat{\mathrm{II}}) \partial \widehat{\Omega}_{k} \cap B\left(y, 10 r^{\prime}\right)=\left\{\left(x^{\prime}, \psi\left(x^{\prime}\right)\right)\right\} \cap B\left(y, 10 r^{\prime}\right), \\
& \widehat{\mathrm{III}}) \widehat{\Omega}_{k} \cap B\left(y, 10 r^{\prime}\right)=\left\{\left(x^{\prime}, x_{n}\right): x_{n}>\psi\left(x^{\prime}\right)\right\} \cap B\left(y, 10 r^{\prime}\right),
\end{aligned}
$$

whenever $y \in L_{k}$. Next we define $\widetilde{\Omega}_{k} \supseteq \widehat{\Omega}_{p}, 1 \leq k \leq p$, relative to $\widehat{\Omega}_{k}$ in the same way that $\Omega^{\prime}$ was defined by adding spherical bumps to $\Omega$. 
That is,

$\widetilde{I}) \widehat{\Omega}_{k} \backslash\left(\bigcup_{z \in L_{k}} B\left(z, \sigma_{j}^{2} r_{j}\right)\right)=\widetilde{\Omega}_{k} \backslash\left(\bigcup_{z \in L_{k}} B\left(z, \sigma_{j}^{2} r_{j}\right)\right)$,

$\widetilde{I I}) \partial \widetilde{\Omega}_{k} \cap \bar{B}\left(y, \sigma_{j}^{2} r_{j}\right)=\bar{B}\left(y, \sigma_{j}^{2} r_{j}\right) \cap\left(\partial \widehat{\Omega}_{k} \backslash B(\widetilde{y}, \widetilde{r}) \cup S(\widetilde{y}, \widetilde{r}) \backslash \widehat{\Omega}_{k}\right)$,

whenever $y \in L_{k}$,

$\widetilde{I I I}) \widetilde{\Omega}_{k} \cap B\left(y, \sigma_{j}^{2} r_{j}\right)=\left(B(\widetilde{y}, \widetilde{r}) \cup \widehat{\Omega}_{k}\right) \cap B\left(y, \sigma_{j}^{2} r_{j}\right)$ whenever $y \in L_{k}$.

Here $\widetilde{y}, \widetilde{r}$ are defined as in (2.10) relative to $y$. From the definition of $\Omega, \Omega^{\prime}$ we see that $\widehat{\Omega}_{k} \supseteq \Omega, \widetilde{\Omega}_{p} \supseteq \Omega^{\prime}$ for $1 \leq k \leq p$. Also from the definition of $\psi$ and (2.5) it can be shown as in [LV, Section 4] that $\widehat{\Omega}_{k}, \widetilde{\Omega}_{k}, 1 \leq k \leq p$, are NTA and Lipschitz on scale $r^{\prime}$ with constant 1000. Let $\widehat{\Omega}_{0}=\Omega, \widetilde{\Omega}_{0}=\widehat{\Omega}_{p}$. From the defininion of $\left\{\lambda_{k}\right\}$ and our restriction on $\sigma_{0}$ we deduce after a possible rotation and translation that Lemma 4.19 can be applied with $\Omega_{1}=\widetilde{\Omega}_{0}, \Omega_{2}=\widetilde{\Omega}_{1}$. Next by the same reasoning we can apply Lemma 4.19 with $\Omega_{1}=\widetilde{\Omega}_{1}$ and $\Omega_{2}=$ $\widetilde{\Omega}_{2}, \ldots$, etc. Let $\widehat{G}_{k}, \widetilde{G}_{k}, \widehat{\omega}_{k}, \widetilde{\omega}_{k}$, be the Green's functions and harmonic measures relative to 0 for $\widehat{\Omega}_{k}, \widetilde{\Omega}_{k}$. Applying the above argument $p$ times we obtain an inequality for $\widehat{G}_{p}=\widetilde{G}_{0}$ and $\widetilde{G}_{p}$. Using the definition of $\left\{\lambda_{k}\right\}_{0}^{\infty}$, we conclude

$$
\begin{aligned}
\int_{\partial \widetilde{\Omega}_{p}}\left|\nabla \widetilde{G}_{p}\right| \log \left|\nabla \widetilde{G}_{p}\right| d H^{n-1} \\
\leq \int_{\partial \widehat{\Omega}_{p}}\left|\nabla \widehat{G}_{p}\right| \log \left|\nabla \widehat{G}_{p}\right| d H^{n-1} \\
\\
\quad-c(n)\left(\lambda_{l+1}\right)^{-(n-1)} \sum_{k=0}^{p-1} \widetilde{\omega}_{k}\left(B\left(z_{k+1}, 2 r^{\prime}\right)\right) .
\end{aligned}
$$

To prove (3.14) we must show that $\widehat{G}_{p}, \widetilde{G}_{p}$, in (5.1) can be replaced by $G, G^{\prime}$, with a manageable error term. To do so we introduce $\Omega_{k}^{\prime}, 0 \leq$ $k \leq p$, defined by $\Omega_{0}^{\prime}=\Omega^{\prime}$, and for $1 \leq k \leq p$,

$$
\begin{aligned}
& \left.\mathrm{I}^{\prime}\right) \Omega_{k}^{\prime} \backslash\left(\bigcup_{z \in L_{k}} B\left(z, 10 r^{\prime}\right)\right)=\Omega^{\prime} \backslash\left(\bigcup_{z \in L_{k}} B\left(z, 10 r^{\prime}\right)\right), \\
& \left.\mathrm{II}^{\prime}\right) \partial \Omega_{k}^{\prime} \cap B\left(y, 10 r^{\prime}\right)=\partial \widetilde{\Omega}_{k} \cap B\left(y, 10 r^{\prime}\right), \\
& \left.\mathrm{III}^{\prime}\right) \Omega_{k}^{\prime} \cap B\left(y, 10 r^{\prime}\right)=\widetilde{\Omega}_{k} \cap B\left(y, 10 r^{\prime}\right),
\end{aligned}
$$


for each $y \in L_{k}$. Denote the corresponding Green's functions and harmonic measures relative to 0 , by $G_{k}^{\prime}, \omega_{k}^{\prime}, 1 \leq k \leq p$. We shall also need the following facts about the NTA domain $\Omega_{1}$ with constant 1000 satisfying (4.18). If $z \in \partial \Omega_{1}$ and $0<\rho \leq 10$, then

$$
\begin{aligned}
\omega_{1}(B(z, \rho)) & \leq c(n) \rho^{n-2} \max _{B(z, \rho) \cap \Omega_{1}} G_{1} \\
& \leq c(n) \rho^{n-2} G_{1}\left(P_{\rho}\right) \leq c(n) \omega_{1}(B(z, \rho)),
\end{aligned}
$$

where $P_{\rho}=P_{\rho}(z)$. Moreover,

$$
\omega_{1}(B(z, 2 \rho)) \leq c(n) \omega_{1}(B(z, \rho)) .
$$

(5.3) is called the doubling inequality for harmonic measure. Also, there exists $\mu=\mu(A)>0$ so that for $z, P_{\rho}$, as above, and $x \in B(z, \rho) \cap \Omega_{1}$,

$$
G_{1}(x) \leq c(n)\left(\frac{|x-z|}{\rho}\right)^{\mu} G_{1}\left(P_{\rho}\right) .
$$

From Harnack's inequality, it follows that there exists $\nu=\nu(n), 1<$ $\nu<\infty$, with

$$
c(n)^{-1} \rho^{\nu} \leq \omega_{1}(B(z, \rho)) \leq 1, \quad 0<\rho \leq 1 .
$$

Next we note that if $z \in \partial \Omega_{1}$ and $u, v$, are two positive harmonic functions in $\Omega_{1}$ which vanish continuously on $\partial \Omega_{1} \backslash B(z, \rho)$, and $P_{\rho}=$ $P_{\rho}(z)$, then for $x \in \Omega_{1} \backslash B(z, 2 \rho)$

$$
c(n)^{-1} \frac{u\left(P_{\rho}\right)}{v\left(P_{\rho}\right)} \leq \frac{u(x)}{v(x)} \leq c(n) \frac{u\left(P_{\rho}\right)}{v\left(P_{\rho}\right)} .
$$

Moreover, (5.6) is valid when $u, v$, vanish on $\partial \Omega_{1} \cap B(z, 2 \rho)$, and $x \in B(z, \rho) \cap \Omega_{1}$. (5.6) is called the rate inequality. Next since $\Omega_{1}$ is Lipschitz on scale $t$, we have for $0<t_{1} \leq t$,

$$
t_{1}^{1-n} \int_{B\left(z, t_{1}\right)}\left|\nabla G_{1}\right|^{2} d H^{n-1} \leq c(n)\left(t_{1}{ }^{1-n} \omega_{1}\left(B\left(z, t_{1}\right)\right)\right)^{2},
$$

which is called an $L^{2}$ reverse Hölder inequality. Using (5.7) and Hölder's inequality one easily deduces the following $A_{\infty}$ type condition. If $E \subset$ $B\left(z, t_{1}\right)$ is a Borel set, then

$$
\frac{\omega_{1}(E)}{\omega_{1}\left(B\left(z, t_{1}\right)\right)} \leq c(n)\left(\frac{H^{n-1}(E)}{H^{n-1}\left(B\left(z, t_{1}\right)\right)}\right)^{1 / 2} .
$$


Also using (5.5), (5.7) and Jensen's inequality one deduces,

$$
\int_{B\left(z, t_{1}\right)}\left|\nabla G_{1}\right||\log | \nabla G_{1}|| d H^{n-1} \leq-c(n) \log t_{1} \omega_{1}\left(B\left(z, t_{1}\right)\right) .
$$

For the proof of (5.2)-(5.6) see [JK, sections 4 and 5]. (5.7) follows from (5.6) and a result of Dahlberg (see [D]). Using (5.2)-(5.5) it follows as in $[\mathrm{LV},(3.10)]$ that

$$
\sum_{k=0}^{p-1} \omega_{k}^{*}\left(B\left(z_{k+1}, 6 r^{\prime}\right)\right) \leq c(n)
$$

whenever $*$ is an element of $\left\{\wedge, \sim,^{\prime}\right\}$. We show for $0 \leq k \leq p-1$ that

$$
\begin{aligned}
\int_{\partial \Omega_{k}^{\prime}}\left|\nabla G_{k}^{\prime}\right| \log \left|\nabla G_{k}^{\prime}\right| d H^{n-1} \leq & \int_{\partial \Omega_{k+1}^{\prime}}\left|\nabla G_{k+1}^{\prime}\right| \log \left|\nabla G_{k+1}^{\prime}\right| d H^{n-1} \\
& +c(n)\left(r^{\prime}\right)^{1 / 8} \omega_{k}^{\prime}\left(B\left(z_{k+1}, 3 r^{\prime}\right)\right),
\end{aligned}
$$

$$
\begin{aligned}
\int_{\partial \widehat{\Omega}_{k+1}}\left|\nabla \widehat{G}_{k+1}\right| \log \left|\nabla \widehat{G}_{k+1}\right| d H^{n-1} \leq & \int_{\partial \widehat{\Omega}_{k}}\left|\nabla \widehat{G}_{k}\right| \log \left|\nabla \widehat{G}_{k}\right| d H^{n-1} \\
& +c(n)\left(r^{\prime}\right)^{1 / 8} \widehat{\omega}_{k}\left(B\left(z_{k+1}, 3 r^{\prime}\right)\right) .
\end{aligned}
$$

Summing (5.11) and using (5.10), it then follows that

$$
\begin{aligned}
\int_{\partial \Omega^{\prime}}\left|\nabla G^{\prime}\right| \log \mid & \nabla G^{\prime} \mid d H^{n-1} \\
& \leq \int_{\partial \widetilde{\Omega}_{p}}\left|\nabla \widetilde{G}_{p}\right| \log \left|\nabla \widetilde{G}_{p}\right| d H^{n-1}+c(n)\left(r^{\prime}\right)^{1 / 8}
\end{aligned}
$$

where we have used the fact that $\Omega_{0}^{\prime}=\Omega^{\prime}, \Omega_{p}^{\prime}=\widetilde{\Omega}_{p}$. Summing (5.12) and using (5.10), we find

$$
\begin{aligned}
\int_{\partial \widehat{\Omega}_{p}}\left|\nabla \widehat{G}_{p}\right| \log \mid & \nabla \widehat{G}_{p} \mid d H^{n-1} \\
\leq & \int_{\partial \Omega}|\nabla G| \log |\nabla G| d H^{n-1}+c(n)\left(r^{\prime}\right)^{1 / 8}
\end{aligned}
$$


since $\widehat{\Omega}_{0}=\Omega$. Putting (5.13), (5.14) into (5.1) we get

$$
\begin{aligned}
\int_{\partial \Omega^{\prime}}\left|\nabla G^{\prime}\right| \log \mid & \nabla G^{\prime} \mid d H^{n-1} \\
& \leq \int_{\partial \Omega}|\nabla G| \log |\nabla G| d H^{n-1}+c(n)\left(r^{\prime}\right)^{1 / 8}
\end{aligned}
$$

Using this inequality in the definition of $D_{m+1}$ we obtain

$$
\begin{aligned}
& \int_{\partial D_{m+1}}\left|\nabla G_{m+1}\right| \log \left|\nabla G_{m+1}\right| d H^{n-1} \\
& \leq \int_{\partial D_{m}}\left|\nabla G_{m}\right| \log \left|\nabla G_{m}\right| d H^{n-1}+c(n)\left(r^{\prime}\right)^{1 / 8},
\end{aligned}
$$

where $r^{\prime}=r^{\prime}(m)$. From (2.5) and the definition of $\delta_{k}$ following (3.9) we see that $\sum_{m=0}^{\infty}\left(r^{\prime}(m)\right)^{1 / 8}<\infty$. Hence (3.14) is true and the proof of Theorem 1 is complete after we prove (5.11), (5.12).

We prove only (5.11) for $k=0$, since the proof of all the other inequalities is the same. To prove (5.11) for $k=0$ let $y=z_{1}$ in the definition of $\Omega_{1}^{\prime}$ and let $\psi$ be as defined earlier relative to $y$. If $\widetilde{y}, \widetilde{r}$ are as in (2.10), put

$$
\begin{aligned}
& \tau\left(x^{\prime}\right) \\
& \quad= \begin{cases}\min \left\{\psi\left(x^{\prime}\right), \widetilde{y}_{n}-\sqrt{(\widetilde{r})^{2}-\left|w^{\prime}-x^{\prime}\right|^{2}}\right\}, & \text { for }\left|x^{\prime}-w^{\prime}\right| \leq \widetilde{r}, \\
\psi\left(x^{\prime}\right), & \text { for } \widetilde{r} \leq\left|x^{\prime}-w^{\prime}\right| \leq \rho^{*}\end{cases}
\end{aligned}
$$

Then

$$
\begin{gathered}
\partial \Omega_{1}^{\prime} \cap B\left(y, 10 r^{\prime}\right)=\left\{\left(x^{\prime}, \tau\left(x^{\prime}\right)\right)\right\} \cap B\left(y, 10 r^{\prime}\right), \\
\Omega_{1}^{\prime} \cap B\left(y, 10 r^{\prime}\right)=\left\{\left(x^{\prime}, x_{n}\right): x_{n}>\tau\left(x^{\prime}\right)\right\} \cap B\left(y, 10 r^{\prime}\right) .
\end{gathered}
$$

Also if

$$
\sigma\left(x^{\prime}\right)=\left\{\begin{array}{l}
\min \left\{w_{n}-\sqrt{\left(\rho^{*}\right)^{2}-\left|x^{\prime}-w^{\prime}\right|^{2}}, \widetilde{y}_{n}-\sqrt{(\widetilde{r})^{2}-\left|x^{\prime}-w^{\prime}\right|^{2}}\right\}, \\
\text { for }\left|x^{\prime}-w^{\prime}\right| \leq \widetilde{r} \\
w_{n}-\sqrt{\left(\rho^{*}\right)^{2}-\left|x^{\prime}-w^{\prime}\right|^{2}} \\
\text { for } \widetilde{r} \leq\left|x^{\prime}-w^{\prime}\right| \leq \rho^{*}
\end{array}\right.
$$


then

$$
\begin{gathered}
\partial \Omega^{\prime} \cap B\left(y, 10 r^{\prime}\right)=\left\{\left(x^{\prime}, \sigma\left(x^{\prime}\right)\right)\right\} \cap B\left(y, 10 r^{\prime}\right) \\
\Omega^{\prime} \cap B\left(y, 10 r^{\prime}\right)=\left\{\left(x^{\prime}, x_{n}\right): x_{n}>\sigma\left(x^{\prime}\right)\right\} \cap B\left(y, 10 r^{\prime}\right) .
\end{gathered}
$$

Next let

$$
\begin{gathered}
K_{1}=\left\{x^{\prime}:\left(x^{\prime}, x_{n}\right) \in S(\widetilde{y}, \widetilde{r}) \cap S\left(w, \rho^{*}\right)\right\}, \\
K_{2}=\left\{x^{\prime}:\left(x^{\prime}, w_{n}-\rho^{*}\right) \in S(\widetilde{y}, \widetilde{r})\right\}, \\
K_{3}=\left\{x^{\prime}: r^{\prime} \leq\left|x^{\prime}-w^{\prime}\right| \leq r^{\prime}+\left(r^{\prime}\right)^{3 / 2}\right\} .
\end{gathered}
$$

Let $K$ be the set of all $x^{\prime} \in \mathbb{R}^{n-1}$ whose distance from $\cup_{i=1}^{3} K_{i}$ is at most $1000\left(r^{\prime}\right)^{3 / 2}$ and set

$$
\begin{gathered}
H=\left\{x^{\prime}:\left|x^{\prime}-w^{\prime}\right|<3 r^{\prime}\right\} \backslash K, \\
K^{\prime}=\left\{\left(x^{\prime}, x_{n}\right) \in \partial \Omega^{\prime} \cap B\left(z_{1}, 3 r^{\prime}\right): x^{\prime} \in K\right\}, \\
K_{1}^{\prime}=\left\{\left(x^{\prime}, x_{n}\right) \in \partial \Omega_{1}^{\prime} \cap B\left(z_{1}, 3 r^{\prime}\right): x^{\prime} \in K\right\} .
\end{gathered}
$$

We have

$$
\begin{aligned}
& \left|\int_{\partial \Omega^{\prime} \cap B\left(z_{1}, 3 r^{\prime}\right)}\right| \nabla G^{\prime}|\log | \nabla G^{\prime} \mid d H^{n-1} \\
& \quad-\int_{\partial \Omega_{1}^{\prime} \cap B\left(z_{1}, 3 r^{\prime}\right)}\left|\nabla G_{1}^{\prime}\right| \log \left|\nabla G_{1}^{\prime}\right| d H^{n-1} \mid \\
& \leq\left|\int_{K^{\prime}}\right| \nabla G^{\prime}|\log | \nabla G^{\prime}\left|d H^{n-1}-\int_{K_{1}^{\prime}}\right| \nabla G_{1}^{\prime}|\log | \nabla G_{1}^{\prime}\left|d H^{n-1}\right| \\
& \quad+\left|\int_{\left(\partial \Omega^{\prime} \backslash K^{\prime}\right) \cap B\left(z_{1}, 3 r^{\prime}\right)}\right| \nabla G^{\prime}|\log | \nabla G^{\prime} \mid d H^{n-1} \\
& \quad-\int_{\left(\partial \Omega_{1}^{\prime} \backslash K_{1}^{\prime}\right) \cap B\left(z_{1}, 3 r^{\prime}\right)}\left|\nabla G_{1}^{\prime}\right| \log \left|\nabla G_{1}^{\prime}\right| d H^{n-1} \mid \\
& =T_{1}+T_{2} .
\end{aligned}
$$

To estimate $T_{1}$ we cover $K^{\prime}$ by balls of radius $10\left(r^{\prime}\right)^{3 / 2}$ with centers in $K^{\prime}$ and the property that the balls with the same centers and radius 
$\left(r^{\prime}\right)^{3 / 2}$ are disjoint. Using (5.9) in each ball and (5.3), (5.8) with $E=$ $K^{\prime}, t_{1}=3 r^{\prime}$ we deduce after summing that

$$
\begin{aligned}
\left|\int_{K^{\prime} \cap B\left(z_{1}, 3 r^{\prime}\right)}\right| \nabla G^{\prime}|\log | \nabla G^{\prime}\left|d H^{n-1}\right| & \leq-c(n) \log r^{\prime} \omega^{\prime}\left(K^{\prime}\right) \\
& \leq c(n)\left(r^{\prime}\right)^{1 / 8} \omega^{\prime}\left(B\left(z_{1}, 3 r^{\prime}\right)\right) .
\end{aligned}
$$

This inequality also holds with $K^{\prime}, G^{\prime}, \omega^{\prime}$ replaced by $K_{1}^{\prime}, G_{1}^{\prime}, \omega_{1}^{\prime}$. Next we observe that it follows in the same way as (5.10) that $\omega_{1}^{\prime}\left(B\left(z_{1}, 3 r^{\prime}\right)\right)$ $\approx \omega^{\prime}\left(B\left(z_{1}, 3 r^{\prime}\right)\right)$ where $\approx$ means the two quantities are constant multiples of each other (depending only on $n$ ). From the above inequalities we conclude

$$
T_{1} \leq c(n)\left(r^{\prime}\right)^{1 / 8} \omega^{\prime}\left(B\left(z_{1}, 3 r^{\prime}\right)\right)
$$

To begin the estimate of $T_{2}$ we write $x$ for $\left(x^{\prime}, \sigma\left(x^{\prime}\right)\right)$ and $\widehat{x}$ for $\left(x^{\prime}, \tau\left(x^{\prime}\right)\right)$ in the following integrals.

$$
\begin{aligned}
T_{3}= & \left|\int_{\left(\partial \Omega^{\prime} \backslash K^{\prime}\right) \cap B\left(z_{1}, 3 r^{\prime}\right)}\right| \nabla G_{1}^{\prime}|\log | \nabla G_{1}^{\prime} \mid d H^{n-1} \\
& \quad-\int_{\left(\partial \Omega_{1}^{\prime} \backslash K_{1}^{\prime}\right) \cap B\left(z_{1}, 3 r^{\prime}\right)}\left|\nabla G_{1}^{\prime}\right| \log \left|\nabla G_{1}^{\prime}\right| d H^{n-1} \mid \\
\leq & \int_{H}|| \nabla G_{1}^{\prime}|(x) \log | \nabla G_{1}^{\prime}||(x) \mid \\
& \quad \cdot \sqrt{1+\left|\nabla \sigma\left(x^{\prime}\right)\right|^{2}}-\sqrt{1+\left|\nabla \tau\left(x^{\prime}\right)\right|^{2}} \mid d x^{\prime} \\
& +\int_{H}|| \nabla G_{1}^{\prime}|(\widehat{x})-| \nabla G_{1}^{\prime}|(x)||\log | \nabla G_{1}^{\prime}||(x) \sqrt{1+\left|\nabla \tau\left(x^{\prime}\right)\right|^{2}} d x^{\prime} \\
& +\int_{H}\left|\nabla G_{1}^{\prime}\right|(\widehat{x})|\log | \nabla G_{1}^{\prime}(x)|-\log | \nabla G_{1}^{\prime}(\widehat{x})|| \sqrt{1+\left|\nabla \tau\left(x^{\prime}\right)\right|^{2}} d x^{\prime} \\
= & U_{1}+U_{2}+U_{3} .
\end{aligned}
$$

From the definition of $\tau, \sigma$, and (5.9) we find that

$$
U_{1} \leq c(n)\left(r^{\prime}\right)^{1 / 8} \omega_{1}^{\prime}\left(B\left(z_{1}, 3 r^{\prime}\right)\right) \leq c(n)\left(r^{\prime}\right)^{1 / 8} \omega^{\prime}\left(B\left(z_{1}, 3 r^{\prime}\right)\right)
$$

To estimate $U_{2}, U_{3}$ let $\widehat{x}=\left(x^{\prime}, \tau\left(x^{\prime}\right)\right), x=\left(x^{\prime}, \sigma\left(x^{\prime}\right)\right), x^{\prime} \in H$, be as in (5.19). Then $\widehat{x} \in \partial \Omega_{1}^{\prime} \backslash K_{1}^{\prime}$ and using the Kelvin transformation it is 
easily seen that $G_{1}^{\prime}$ extends to a harmonic function in $B\left(\widehat{x}, 2\left(r^{\prime}\right)^{3 / 2}\right)$. If $\rho=\left(r^{\prime}\right)^{3 / 2}$, then from standard estimates for harmonic functions in balls, (5.2), and the fact that $|x-\widehat{x}| \leq c(n)\left(r^{\prime}\right)^{2} / \rho^{*}$, we obtain

$$
\begin{aligned}
\left|\nabla G_{1}^{\prime}(x)-\nabla G_{1}^{\prime}(\widehat{x})\right| & \leq c(n)|x-\widehat{x}| \rho^{-2} \max _{B(\widehat{x}, \rho)} G_{1}^{\prime} \\
& \leq c(n)\left(r^{\prime} \rho^{*}\right)^{-1} G_{1}^{\prime}\left(P_{\rho}(\widehat{x})\right) \\
& \leq c(n)\left(r^{\prime} \rho^{*}\right)^{-1} \rho^{2-n} \omega_{1}^{\prime}(B(\widehat{x}, \rho)) .
\end{aligned}
$$

Using positivity of $G_{1}^{\prime}$ and (5.3) we also find that

$$
c(n)^{-1} \rho^{1-n} \omega_{1}^{\prime}(B(\widehat{x}, \rho)) \leq\left|\nabla G_{1}^{\prime}(\widehat{x})\right| \leq c(n) \rho^{1-n} \omega_{1}^{\prime}(B(\widehat{x}, \rho)) .
$$

Putting (5.22) in (5.21) we find in view of (2.5) that

$$
\left|\nabla G_{1}^{\prime}(x)-\nabla G_{1}^{\prime}(\widehat{x})\right| \leq c(n)\left(r^{\prime}\right)^{1 / 4}\left|\nabla G_{1}^{\prime}\right|(\bar{x}),
$$

where $\bar{x}=x$ or $\widehat{x}$. From (5.23) and (5.9) we see that

$$
U_{2}+U_{3} \leq c(n)\left(r^{\prime}\right)^{1 / 8} \omega^{\prime}\left(B\left(z_{1}, 3 r^{\prime}\right)\right)
$$

Using (5.24), (5.20) in (5.19) we deduce

$$
T_{3} \leq c(n)\left(r^{\prime}\right)^{1 / 8} \omega^{\prime}\left(B\left(z_{1}, 3 r^{\prime}\right)\right) .
$$

If $x \in \partial \Omega^{\prime} \backslash K^{\prime}, \widehat{x}$, and $\rho=\left(r^{\prime}\right)^{3 / 2}$, are as above, then again using the Kelvin transformation we deduce first that $G^{\prime}-G_{1}^{\prime}$ has a harmonic extension to $B(x, 2 \rho)$ and second that

$$
\left|\nabla G^{\prime}-\nabla G_{1}^{\prime}\right|(x) \leq c(n) \rho^{-1} \max _{B(x, \rho)}\left|G^{\prime}-G_{1}^{\prime}\right| .
$$

We claim that

$$
\begin{aligned}
\max _{B(x, \rho)}\left|G^{\prime}-G_{1}^{\prime}\right| & \leq c(n) \max _{B(x, \rho) \cap \Omega^{\prime}}\left|G^{\prime}-G_{1}^{\prime}\right|+c(n) \frac{\left(r^{\prime}\right)^{1 / 2}}{\rho^{*}} G^{\prime}\left(P_{\rho}(x)\right) \\
(5.27) & \leq c(n) \max _{B(x, \rho) \cap \Omega^{\prime}}\left|G^{\prime}-G_{1}^{\prime}\right|+c(n)\left(\left(r^{\prime}\right)^{1 / 2} \frac{\rho}{\rho^{*}}\right)\left|\nabla G^{\prime}\right|(x) .
\end{aligned}
$$

The second line of (5.27) follows from the first line, (5.2), (5.3), and the same argument as in (5.22). To prove the first line of (5.27) observe that if $x \in S(\widetilde{y}, \widetilde{r}) \cap K^{\prime}$, then this inequality is obvious since both functions 
are extended by essentially reflecting across $S(\widetilde{y}, \widetilde{r})$. Otherwise suppose $\bar{z} \in B(x, \rho)$ and $\widetilde{z}, \widehat{z}$ denote the reflection of $\bar{z}$ with respect to the plane $\left\{u \in \mathbb{R}^{n}: u_{n}=w_{n}-\rho^{*}\right\}$ and the sphere $S\left(w, \rho^{*}\right)$, respectively. Then

$$
|\widetilde{z}-\widehat{z}| \leq c(n)\left(r^{\prime}\right)^{1 / 2} \frac{\rho}{\rho^{*}}
$$

Using this fact, the definition of the Kelvin transformation, and standard estimates for functions vanishing on $B(x, \rho) \cap S\left(w, \rho^{*}\right)$ we obtain (5.27). As noted earlier we have

$$
\omega^{\prime}\left(B\left(z_{1}, 3 r^{\prime}\right)\right) \approx \omega_{1}^{\prime}\left(B\left(z_{1}, 3 r^{\prime}\right)\right)
$$

so from (5.2), we have

$$
G^{\prime}\left(P_{r^{\prime}}(x)\right) \approx G_{1}^{\prime}\left(P_{r^{\prime}}(x)\right)
$$

Now if $\sigma_{0}=\sigma_{0}(n)>0$ is small enough, then from this note and a barrier type estimate using interior and exterior cones, we deduce for $r^{\prime} \geq t \geq 2|x-\widehat{x}|$ that

$$
\begin{aligned}
c(n)^{-1}\left(\frac{t}{r^{\prime}}\right)^{11 / 10} \max \left\{G^{\prime}(\right. & \left.\left.P_{r^{\prime}}(x)\right), G_{1}^{\prime}\left(P_{r^{\prime}}(x)\right)\right\} \\
& \leq \min \left\{G^{\prime}\left(P_{t}(x)\right), G_{1}^{\prime}\left(P_{t}(x)\right)\right\}
\end{aligned}
$$

and

$$
\begin{aligned}
& \max \left\{G^{\prime}\left(P_{t}(x)\right), G_{1}^{\prime}\left(P_{t}(x)\right)\right\} \\
& \quad \leq c(n)\left(\frac{t}{r^{\prime}}\right)^{9 / 10} \min \left\{G^{\prime}\left(P_{r^{\prime}}(x)\right), G_{1}^{\prime}\left(P_{r^{\prime}}(x)\right)\right\} .
\end{aligned}
$$

We observe that every point of $\partial \Omega^{\prime} \cap B\left(z_{1}, 3 r^{\prime}\right)$ lies within $c(n)\left(r^{\prime}\right)^{2} / \rho^{*}$ of a point of $\partial \Omega_{1}^{\prime}$. From this observation, the maximum principle for harmonic functions, (5.28) and (2.5) we see that

$$
\begin{aligned}
\max _{B(x, \rho) \cap \Omega^{\prime}}\left|G^{\prime}-G_{1}^{\prime}\right| & \leq c(n) \max _{\partial \Omega^{\prime} \cap B\left(z_{1}, 3 r^{\prime}\right)}\left|G_{1}^{\prime}\right| \\
& \leq c(n)\left(\frac{r^{\prime}}{\rho^{*}}\right)^{9 / 10} G^{\prime}\left(P_{r^{\prime}}(x)\right) \\
& \leq c(n)\left(r^{\prime}\right)^{1 / 4} G^{\prime}\left(P_{\rho}(x)\right) \\
& \leq c(n)\left(r^{\prime}\right)^{1 / 4} \rho\left|\nabla G^{\prime}(x)\right| .
\end{aligned}
$$


Using (5.27), (5.29) in (5.26) we get

$$
\left|\nabla G^{\prime}-\nabla G_{1}^{\prime}\right|(x) \leq c(n)\left(r^{\prime}\right)^{1 / 4}\left|\nabla G^{\prime}(x)\right| .
$$

Finally from (5.30), (5.25) we conclude that

$$
\begin{aligned}
T_{2} \leq & \left|\int_{\left(\partial \Omega^{\prime} \backslash K^{\prime}\right) \cap B\left(z_{1}, 3 r^{\prime}\right)}\right| \nabla G_{1}^{\prime}|\log | \nabla G_{1}^{\prime} \mid d H^{n-1} \\
& -\int_{\left(\partial \Omega^{\prime} \backslash K^{\prime}\right) \cap B\left(z_{1}, 3 r^{\prime}\right)}\left|\nabla G^{\prime}\right| \log \left|\nabla G^{\prime}\right| d H^{n-1} \mid+T_{3} \\
\leq & \int_{H}|| \nabla G_{1}^{\prime}|(x)-| \nabla G^{\prime}|(x)||\log | \nabla G_{1}^{\prime}||(x) \sqrt{1+\left|\nabla \sigma\left(x^{\prime}\right)\right|^{2}} d x^{\prime} \\
& +c(n)\left(r^{\prime}\right)^{1 / 8} \omega^{\prime}\left(B\left(z_{1}, 3 r^{\prime}\right)\right) \\
& +\int_{H}\left|\nabla G^{\prime}\right|(x)|\log | \nabla G_{1}^{\prime}(x)|-\log | \nabla G^{\prime}(x)|| \sqrt{1+\left|\nabla \sigma\left(x^{\prime}\right)\right|^{2}} d x^{\prime} \\
\leq & c(n)\left(r^{\prime}\right)^{1 / 8} \omega^{\prime}\left(B\left(z_{1}, 3 r^{\prime}\right)\right) .
\end{aligned}
$$

From (5.18), (5.31) and (5.17) we conclude that

$$
\begin{aligned}
& \left|\int_{\partial \Omega_{1}^{\prime} \cap B\left(z_{1}, 3 r^{\prime}\right)}\right| \nabla G_{1}^{\prime}|\log | \nabla G_{1}^{\prime} \mid d H^{n-1} \\
& -\int_{\partial \Omega^{\prime} \cap B\left(z_{1}, 3 r^{\prime}\right)}\left|\nabla G^{\prime}\right| \log \left|\nabla G^{\prime}\right| d H^{n-1} \mid \\
& \leq c(n)\left(r^{\prime}\right)^{1 / 8} \omega^{\prime}\left(B\left(z_{1}, 3 r^{\prime}\right)\right) .
\end{aligned}
$$

Next we note that the argument in [LV] from (3.26) to (3.28) uses only NTA estimates (primarily (5.4) and (5.6)) so is also valid for our current domains. Thus

$$
\begin{aligned}
& \left|\int_{\partial \Omega_{1}^{\prime} \backslash B\left(z_{1}, 3 r^{\prime}\right)}\right| \nabla G_{1}^{\prime}|\log | \nabla G_{1}^{\prime} \mid d H^{n-1} \\
& -\int_{\partial \Omega^{\prime} \backslash B\left(z_{1}, 3 r^{\prime}\right)}\left|\nabla G^{\prime}\right| \log \left|\nabla G^{\prime}\right| d H^{n-1} \mid \\
& \quad \leq c(n)\left(r^{\prime}\right)^{1 / 8} \omega^{\prime}\left(B\left(z_{1}, 3 r^{\prime}\right)\right) .
\end{aligned}
$$


From (5.32), (5.33) we find that (5.11) is valid for $k=0$. Fix $\sigma_{0}=\sigma_{0}(n)>0$ subject to the stipulations in sections 2-5. From our earlier remarks we conclude first (3.14) and thereupon that Theorem 1 is valid.

\section{References.}

[A] Ahlfors, L., Quasi conformal Mappings. Van Nostrand, 1966.

[D] Dahlberg, B., On estimates of harmonic measure. Arch. Rational Mech. Anal. 65 (1977), 275-288.

[DSS] Duren, P., Shapiro, H., Shields, A., Singular measures and domains not of Smirnov type. Duke Math. J. 33 (1966), 247-254.

[Du] Duren, P., Theory of $H^{p}$ spaces, Academic Press, 38 (1970).

[FL] Friedman, A., Littman, W., Functions satisfying the mean value property. Trans. Amer. Math. Soc. 102 (1962), 167-180.

[GE] Gariepy, R., Evans, L., Measure theory and fine properties of functions. Studies in Advanced Mathematics. CRC Press, 1992.

[GT] Gilbarg, D., Trudinger, N., Elliptic partial differential equations of second order. Springer-Verlag, 1977.

[H] Helms, L., Introduction to potential theory. Wiley-Interscience, 1969.

[JK] Jerison, D., Kenig, C., Boundary behavior of harmonics functions in non-tangentially accessible domains. Advances in Math. 46 (1982), 80147.

[KL] Keldysh, M., Lavrentiev, M., Sur la représentation conforme des domain limités par des courbes rectifiables. Ann. Sci. École Norm. Sup. $\mathbf{5 4}$ (1937), 1-38.

[LV] Lewis, J., Vogel, A., On pseudospheres. Revista Mat. Iberoamericana 7 (1991), 25-54.

[LV1] Lewis, J., Vogel, A., On some almost everywhere symmetry theorems. Nonlinear diffusion equations and their equilibrium states. 3 (1992), Birkhäuser, 347-374.

[LVV] Lewis, J., Verchota, G., Vogel, A., On Wolf's Integral. In preparation.

$[\mathrm{P}]$ Privalov, I., Boundary properties of analytic functions. Deutscher Verlag, 1956.

[R] Rickman, S., Quasiregular Mappings, 26 (1991), Springer-Verlag.

[Re] Reshetnyak, Y., Space Mappings with Bounded Distortion. Translation of Mathematical Monographs 73 (1989), American Mathematical Society. 
[S] Shapiro, H., Remarks concerning domains of Smirnov type. Michigan Math. J. 13 (1966), 341-348.

[W] Wolff, T., Counterexamples with harmonic gradients in $\mathbb{R}^{3}$. Essays in honor of Elias M. Stein. Princeton Mathematical Series 42 (1995), 321384 .

Recibido: 23 de julio de 1.999

\author{
Andrew Vogel \\ Department of Mathematics \\ Syracuse University \\ Syracuse, NY 13244, U.S.A. \\ alvogel@syr.edu \\ and \\ John L. Lewis* \\ Department of Mathematics \\ University of Kentucky \\ Lexington, KY 40506-0027, U.S.A. \\ john@ms . uky . edu
}

\footnotetext{
* Supported by an NSF Grant.
} 